\title{
A Robust Test for Weak Instruments
}

\author{
José Luis Montiel OLEA \\ Department of Economics, Harvard University, Cambridge, MA 02138 (montiel@fas.harvard.edu)
}

\section{Carolin Pflueger \\ Department of Finance, Sauder School of Business, University of British Columbia, Vancouver BC V6T 1Z2, Canada (carolin.pflueger@sauder.ubc.ca)}

\begin{abstract}
We develop a test for weak instruments in linear instrumental variables regression that is robust to heteroscedasticity, autocorrelation, and clustering. Our test statistic is a scaled nonrobust first-stage $F$ statistic. Instruments are considered weak when the two-stage least squares or the limited information maximum likelihood Nagar bias is large relative to a benchmark. We apply our procedures to the estimation of the elasticity of intertemporal substitution, where our test cannot reject the null of weak instruments in a larger number of countries than the test proposed by Stock and Yogo in 2005. Supplementary materials for this article are available online.
\end{abstract}

KEY WORDS: Autocorrelation; Clustered; Elasticity of intertemporal substitution; $F$ statistic; Heteroscedasticity.

\section{INTRODUCTION}

This article proposes a simple test for weak instruments that is robust to heteroscedasticity, serial correlation, and clustering. Staiger and Stock (1997) and Stock and Yogo (2005) developed widely used tests for weak instruments under the assumption of conditionally homoscedastic serially uncorrelated model errors. However, applications with heteroscedasticity, time series autocorrelation, and clustered panel data are common. Our proposed test provides empirical researchers with a new tool to assess instrument strength for those applications.

The practical relevance of heteroscedasticity in linear instrumental variable (IV) regression has been highlighted before by Antoine and Lavergne (2012), Chao and Newey (2012), and Hausman et al. (2012). We show, more generally, that departures from the conditionally homoscedastic serially uncorrelated framework affect the weak instrument asymptotic distribution of both the two-stage least squares (TSLS) and the limited information maximum likelihood (LIML) estimators. Consequently, heteroscedasticity, autocorrelation, and/or clustering can further bias estimators and distort test sizes when instruments are potentially weak. At the same time, the first stage may falsely indicate that instruments are strong.

Under strong instruments, both TSLS and LIML are asymptotically unbiased, while such is generally not the case when instruments are weak. We follow the standard Nagar (1959) methodology to derive a tractable proxy for the asymptotic estimator bias that is defined for both TSLS and LIML. Our procedure tests the null hypothesis that the Nagar bias is large relative to a "worst-case" benchmark. Our benchmark coincides with the ordinary least squares (OLS) bias benchmark when the model errors are conditionally homoscedastic and serially uncorrelated, but differs otherwise.

Our proposed test statistic, which we call the effective $F$ statistic, is a scaled version of the nonrobust first-stage $F$ statistic. The null hypothesis for weak instruments is rejected for large values of the effective $F$. The critical values depend on an estimate of the covariance matrix of the OLS reduced form regression coefficients, and on the covariance matrix of the reduced form errors, which can be estimated using standard procedures.

We consider two different testing procedures: generalized and simplified; both are asymptotically valid. Critical values for both procedures can be calculated either by Monte Carlo methods or by a curve-fitting methodology by Patnaik (1949). The generalized testing procedure applies to both TSLS and LIML, and has increased power, but is computationally more demanding. In contrast, the simplified procedure applies only to TSLS. The simplified procedure is conservative, because it protects against the worst type of heteroscedasticity, serial correlation, and/or clustering in the second stage.

Empirical researchers frequently report the robust $F$ statistic as a simple way of adjusting the Staiger and Stock (1997) and Stock and Yogo (2005) pretests for heteroscedasticity, serial correlation, and clustering, and compare them to the homoscedastic critical values. To the best of our knowledge, there is no theoretical or analytical support for this practice, as cautioned in Baum, Schaffer, and Stillman (2007). Our proposed procedures adjust the critical values. While our proposed test statistic corresponds to the robust $F$ statistic in the just identified case, it differs in the overidentified case.

Our baseline implementation tests the null hypothesis that the Nagar bias exceeds $10 \%$ of a"worst-case" bias with a size of 5\%. The simplified procedure for TSLS has critical values between 11 and 23.1 that depend only on the covariance matrix of the first-stage reduced form coefficients. Thus, a simple, asymptotically valid rule of thumb is available for TSLS that rejects when the effective $F$ is greater than 23.1.

We apply weak instrument pretests to a well-known empirical example, the IV estimation of the Elasticity of Intertemporal Substitution (EIS; Campbell 2003; Yogo 2004). Our empirical results are consistent with Yogo's (2004) finding that the EIS

(c) 2013 American Statistical Association Journal of Business \& Economic Statistics July 2013, Vol. 31, No. 3 DOI: 10.1080/00401706.2013.806694 
is small and close to zero. However, for several countries in our sample, conditionally homoscedastic serially uncorrelated pretests indicate strong instruments, while our proposed test cannot reject the null hypothesis of weak instruments.

There is a large literature on inference when IVs are weak; see Stock, Wright, and Yogo (2002), and Andrews and Stock (2006) for overviews. Our article is closest to Staiger and Stock (1997) and Stock and Yogo (2005). Zhan (2010) provided another interesting approach, which, unlike ours, proposes to test the null hypothesis of strong instruments. Bun and de Haan (2010) pointed out the invalidity of pretests based on the firststage $F$ statistic in two particular examples of nonhomoscedastic and serially correlated errors, but do not provide a valid pretest.

Robust methods for inference about the coefficients of a single endogenous regressor when IVs are weak and errors are heteroscedastic and/or serially correlated are also available (Andrews and Stock 2006; Kleibergen 2007). A pretest for weak instruments followed by standard inference procedures can be less computationally demanding, and the use of this twostage decision rule is widespread because of its simplicity. We therefore view this article as complementary to robust inference methods.

It is well known that pretests can induce uniformity problems (Leeb and Poetscher 2005; Guggenberger 2010a,b). However, Stock and Yogo (2005) showed that in the conditionally homoscedastic and serially uncorrelated case the first-stage $F$ statistic can be used to control the Wald test size distortion. In this case, uniformity problems are therefore not a first-order concern.

The rest of the article is organized as follows. Section 2 introduces the model and presents the generalized and simplified testing procedures. Section 3 derives asymptotic distributions and shows that conditional heteroscedasticity and serial correlation can effectively weaken instruments in an illustrative example. Section 4 derives the expressions for the TSLS and LIML Nagar biases and describes the test statistic and critical values. Section 5 discusses the implementation of the critical values by Monte Carlo simulation and Patnaik's (1949) methodology. Section 6 applies the pretesting procedure to the IV estimation of the EIS. Section 7 concludes. All proofs are collected in the Appendix.

\section{MODEL AND SUMMARY OF TESTING PROCEDURE}

\subsection{Model and Assumptions}

We consider a linear IV model in reduced form with one endogenous regressor and $K$ instruments

$$
\begin{aligned}
\mathbf{y} & =\mathbf{Z} \boldsymbol{\Pi} \beta+\mathbf{v}_{1}, \\
\mathbf{Y} & =\mathbf{Z} \boldsymbol{\Pi}+\mathbf{v}_{2} .
\end{aligned}
$$

The structural parameter of interest is $\beta \in \mathbb{R}$, while $\Pi \in \mathbb{R}^{K}$ denotes the unknown first-stage parameter vector. The sample size is $S$ and the econometrician observes the dataset $\left\{y_{s}, Y_{s}, \mathbf{Z}_{s}\right\}_{s=1}^{S}$. We denote the observations of the outcome variable, the endogenous regressor, and the vector of instruments by $y_{s}, Y_{s}$, and $\mathbf{Z}_{s}$, respectively. The unobserved reduced form errors have realiza- tions $v_{j s}, j \in\{1,2\}$. We stack the realized variables in matrices $\mathbf{y} \in \mathbb{R}^{S}, \mathbf{Z} \in \mathbb{R}^{S \times K}$, and $\mathbf{v}_{j} \in \mathbb{R}^{S}, j \in\{1,2\}$.

Our analysis extends straightforwardly to a model with additional exogenous regressors. In the presence of additional exogenous regressors, TSLS and LIML estimators are unchanged if we replace all variables by their projection errors onto those exogenous regressors. TSLS and LIML are also invariant to normalizing the instruments to be orthonormal. We can therefore assume without loss of generality that there are no additional exogenous regressors, and that $\mathbf{Z}^{\prime} \mathbf{Z} / S=\mathbb{I}_{K}$. When implementing the pretest, an applied researcher needs to normalize the data.

We model weak instruments by assuming that the IV firststage relation is local to zero, following the modeling strategy in Staiger and Stock (1997).

Assumption $\mathbf{L}_{\Pi}$ (Local to Zero) $\boldsymbol{\Pi}=\boldsymbol{\Pi}_{S}=\mathbf{C} / \sqrt{S}$, where $\mathbf{C}$ is a fixed vector $\mathbf{C} \in \mathbb{R}^{K}$.

Additional high-level assumptions allow us to derive asymptotic distributions for IV estimators and $F$ statistics. TSLS and LIML estimators and first-stage $F$ statistics depend on the statistics $\mathbf{Z}^{\prime} \mathbf{v}_{j} / \sqrt{S}$, and estimates of the covariance matrices $\mathbf{W}$ and $\boldsymbol{\Omega}$ as defined below.

Assumption HL. (High Level) The following limits hold as $S \rightarrow \infty$.

1. $\left(\begin{array}{l}\mathbf{Z}^{\prime} \mathbf{v}_{1} / \sqrt{S} \\ \mathbf{Z}^{\prime} \mathbf{v}_{2} / \sqrt{S}\end{array}\right) \stackrel{d}{\rightarrow} \mathcal{N}_{2 K}(\mathbf{0}, \mathbf{W})$ for some positive definite $\mathbf{W}=\left(\begin{array}{ll}\mathbf{W}_{1} & \mathbf{W}_{12} \\ \mathbf{W}_{12}^{\prime} & \mathbf{W}_{2}\end{array}\right)$.

2. $\left[\mathbf{v}_{1}, \mathbf{v}_{2}\right]^{\prime}\left[\mathbf{v}_{1}, \mathbf{v}_{2}\right] / S \stackrel{p}{\rightarrow} \boldsymbol{\Omega}$ for some positive definite $\boldsymbol{\Omega} \equiv\left(\begin{array}{cc}\omega_{1}^{2} & \omega_{12} \\ \omega_{12} & \omega_{2}^{2}\end{array}\right)$.

3. There exists a sequence of positive definite estimates $\{\widehat{\mathbf{W}}(S)\}$, measurable with respect to $\left\{y_{s}, Y_{s}, \mathbf{Z}_{s}\right\}_{s=1}^{S}$, such that $\widehat{\mathbf{W}}(S) \stackrel{p}{\rightarrow} \mathbf{W}$ as $S \rightarrow \infty$.

Assumption HL is satisfied under various primitive conditions on the joint distribution of $\left(\mathbf{Z}, \mathbf{v}_{1}, \mathbf{v}_{2}\right)$; see supplementary materials C.2, for examples. Assumption HL.1 is satisfied as long as a central limit theorem holds for $\mathbf{Z}^{\prime} \mathbf{v}_{j} / \sqrt{S}$. Assumption HL.2 holds under a weak law of large numbers for $\left[\mathbf{v}_{1}, \mathbf{v}_{2}\right]^{\prime}\left[\mathbf{v}_{1}, \mathbf{v}_{2}\right] / S$. Assumption HL. 3 assumes that we can consistently estimate the covariance matrix $\mathbf{W}$ from the observable variables.

Assumption HL allows for a general form of $\mathbf{W}$, similarly to the models in Müller (2011) and Mikusheva (2010). This is our key generalization from the model in Staiger and Stock (1997), who required $\mathbf{W}$ to have the form $\Omega \otimes I_{K}$. The Kronecker form arises naturally only in the context of a conditionally homoscedastic serially uncorrelated model. Our generalization is therefore relevant for practitioners working with heteroscedastic, time series, or panel data, and it is consequential for econometric practice.

\subsection{Implementing the Testing Procedure}

2.2.1 Generalized Test. The generalized testing procedure can be implemented in four simple steps. When rejecting the null, the empirical researcher can conclude that the estimator Nagar bias is small relative to the benchmark. Under the null hypothesis, the Nagar bias of TSLS or LIML is greater than a 
fraction $\tau$ of the benchmark. Critical values for the effective $F$ statistic depend on the desired threshold $\tau$, the desired level of significance $\alpha$, and estimates for the matrices $\widehat{\mathbf{W}}, \widehat{\mathbf{\Omega}}$. Critical values also vary between TSLS and LIML. In our numerical results, we focus on $\tau=10 \%$ and $\alpha=5 \%$.

1. If there are additional exogenous regressors, replace all variables by their projection residuals onto those exogenous regressors. Normalize instruments to be orthonormal.

2. Obtain $\widehat{\mathbf{W}}$ as the estimate for the asymptotic covariance matrix of the reduced form OLS coefficients. Standard statistical packages estimate this matrix (divided by the sample size S) under different distributional assumptions. For crosssectionally heteroscedastic applications, use a heteroscedasticity robust estimate; for time series applications, use a heteroscedasticity and autocorrelation consistent (HAC) estimate; and for panel data applications, use a "clustered" estimate.

3. Compute the test statistic, the Effective F Statistic

$$
\widehat{F}_{\text {eff }} \equiv \frac{1}{S} \frac{\mathbf{Y}^{\prime} \mathbf{Z Z} \mathbf{Z}^{\prime} \mathbf{Y}}{\operatorname{tr}\left(\widehat{\mathbf{W}}_{2}\right)}
$$

where $\operatorname{tr}(\cdot)$ denotes the trace operator.

4. Estimate the effective degrees of freedom

$$
\widehat{K}_{\text {eff }} \equiv \frac{\left[\operatorname{tr}\left(\widehat{\mathbf{W}}_{2}\right)\right]^{2}(1+2 x)}{\operatorname{tr}\left(\widehat{\mathbf{W}}_{2}^{\prime} \widehat{\mathbf{W}}_{2}\right)+2 x \operatorname{tr}\left(\widehat{\mathbf{W}}_{2}\right) \max \operatorname{eval}\left(\widehat{\mathbf{W}}_{2}\right)},
$$

where

$$
x=B_{e}(\widehat{\mathbf{W}}, \widehat{\mathbf{\Omega}}) / \tau \text { for } e \in\{\mathrm{TSLS}, \mathrm{LIML}\} .
$$

Here max eval $\left(\widehat{\mathbf{W}}_{2}\right)$ denotes the maximum eigenvalue of the lower diagonal $K \times K$ block of the matrix $\widehat{\mathbf{W}}$. The function $B_{e}(\widehat{\mathbf{W}}, \widehat{\mathbf{\Omega}})$ is closely related to the supremum of the Nagar bias relative to the benchmark; see Theorem 1.2. The numerical implementation of $B_{e}(\widehat{\mathbf{W}}, \widehat{\boldsymbol{\Omega}})$ is discussed in Remark 5, Theorem 1. A fast numerical MATLAB routine is available for the function $B_{e}(\widehat{\mathbf{W}}, \widehat{\mathbf{\Omega}})$.

The generalized test rejects the null hypothesis of weak instruments when $\widehat{F}_{\text {eff }}$ exceeds a critical value that can be obtained by either of the following procedures:

(a) Monte Carlo methods, as described in Section 5.

(b) Patnaik's (1949) curve-fitting methodology; Patnaik critical values obtain as the upper $\alpha$ quantile of $\chi_{\widehat{K}_{\text {eff }}}^{2}\left(x \widehat{K}_{\text {eff }}\right) / \widehat{K}_{\text {eff }}$, where $\chi_{\widehat{K}_{\text {eff }}^{2}}^{2}\left(x \widehat{K}_{\text {eff }}\right)$ denotes a noncentral $\chi^{2}$ distribution with $\widehat{K}_{\text {eff }}$ degrees of freedom and noncentrality parameter $x \widehat{K}_{\text {eff }}$. Table 1 tabulates $5 \%$ Patnaik critical values.

2.2.2 Simplified Test. A simplified conservative version of the test is available for TSLS. The simplified procedure follows the same steps, but sets $x=1 / \tau$ in Step 4. For a given effective degrees of freedom $\widehat{K}_{\text {eff }}$, the simplified $5 \%$ critical value can be conveniently read off Table 1 . For instance, the critical value for a threshold $\tau=10 \%$ can be found in the column with $x=$ 10. The simplified test does not require numerical evaluation of $B_{e}(\widehat{\mathbf{W}}, \widehat{\boldsymbol{\Omega}})$, for it uses the bound $B_{\mathrm{TSLS}}(\widehat{\mathbf{W}}, \widehat{\boldsymbol{\Omega}}) \leq 1$, proved in Theorem 1.3. The matrix $\widehat{\mathbf{W}}$ enters only through the lower

\begin{tabular}{|c|c|c|c|c|}
\hline$K_{\text {eff }}$ & $x=3.33$ & $x=5$ & $x=10$ & $x=20$ \\
\hline 1 & 12.05 & 15.06 & 23.11 & 37.42 \\
\hline 2 & 9.57 & 12.17 & 19.29 & 32.32 \\
\hline 3 & 8.53 & 10.95 & 17.67 & 30.13 \\
\hline 4 & 7.92 & 10.23 & 16.72 & 28.85 \\
\hline 5 & 7.51 & 9.75 & 16.08 & 27.98 \\
\hline 6 & 7.21 & 9.40 & 15.62 & 27.35 \\
\hline 7 & 6.98 & 9.14 & 15.26 & 26.86 \\
\hline 8 & 6.80 & 8.92 & 14.97 & 26.47 \\
\hline 9 & 6.65 & 8.74 & 14.73 & 26.15 \\
\hline 10 & 6.52 & 8.59 & 14.53 & 25.87 \\
\hline 11 & 6.41 & 8.47 & 14.36 & 25.64 \\
\hline 12 & 6.32 & 8.36 & 14.21 & 25.44 \\
\hline 13 & 6.24 & 8.26 & 14.08 & 25.26 \\
\hline 14 & 6.16 & 8.17 & 13.96 & 25.10 \\
\hline 15 & 6.10 & 8.10 & 13.86 & 24.96 \\
\hline 16 & 6.04 & 8.03 & 13.77 & 24.83 \\
\hline 17 & 5.99 & 7.96 & 13.68 & 24.71 \\
\hline 18 & 5.94 & 7.91 & 13.60 & 24.60 \\
\hline 19 & 5.89 & 7.85 & 13.53 & 24.50 \\
\hline 20 & 5.85 & 7.80 & 13.46 & 24.41 \\
\hline 21 & 5.81 & 7.76 & 13.40 & 24.33 \\
\hline 22 & 5.78 & 7.72 & 13.35 & 24.25 \\
\hline 23 & 5.74 & 7.68 & 13.29 & 24.18 \\
\hline 24 & 5.71 & 7.64 & 13.24 & 24.11 \\
\hline 25 & 5.68 & 7.61 & 13.20 & 24.05 \\
\hline 26 & 5.66 & 7.57 & 13.15 & 23.98 \\
\hline 27 & 5.63 & 7.54 & 13.11 & 23.93 \\
\hline 28 & 5.61 & 7.51 & 13.07 & 23.87 \\
\hline 29 & 5.58 & 7.49 & 13.04 & 23.82 \\
\hline 30 & 5.56 & 7.46 & 13.00 & 23.77 \\
\hline
\end{tabular}
$K \times K$ block $\widehat{\mathbf{W}}_{2}$.
Table 1. Critical values: upper $5 \%$ quantile of $\chi_{K_{\text {eff }}}^{2}\left(x K_{\text {eff }}\right) / K_{\text {eff }}$

NOTE: Critical values computed by Patnaik (1949) method. For generalized and simplified testing procedures, estimate $K_{\text {eff }}$ as in Equation (4). For a Nagar bias threshold $\tau$ (e.g., $\tau=10 \%$ ), set $x=1 / \tau$ for the simplified procedure. For the generalized procedure, set $x=B_{e}(\widehat{\mathbf{W}}, \widehat{\boldsymbol{\Omega}}) / \tau ;$ see Step 4 in Section 2.2.1.

\subsubsection{Comparison With Stock and Yogo (2005) Critical} Values. We compare the generalized and simplified TSLS critical values to those proposed by Stock and Yogo (2005) for the case when the data are conditionally homoscedastic and serially uncorrelated. For this comparison, we assume $\mathbf{W}=\boldsymbol{\Omega} \otimes \mathbb{I}_{K}$ and $\mathbf{W}$ and $\boldsymbol{\Omega}$ known, as in Stock and Yogo (2005). It then follows from (3) and (4) that the effective and nonrobust $F$ statistics are equal, and that the effective number of degrees of freedom $K_{\text {eff }}$ equals the number of instruments $K$.

Figure 1 shows the 5\% TSLS critical value for testing the null hypothesis that the asymptotic estimator bias exceeds $10 \%$ of the benchmark, the 5\% critical value for the corresponding simplified test, and the Stock and Yogo (2005) 5\% critical value for testing the null hypothesis that the TSLS bias exceeds $10 \%$ of the OLS bias. The Stock and Yogo (2005) critical value is defined when the degree of overidentification is at least two and we therefore show critical values for $3 \leq K \leq 30$. The TSLS critical value increases from 8.53 for $K=3$ to 12.27 for $K=30$. By comparison, the Stock and Yogo (2005) critical value increases from 9.08 for $K=3$ to 11.32 for $K=30$. The simplified TSLS critical value is strictly larger than the TSLS critical value for all $K$ shown, illustrating that the simplified test can be strictly less powerful than the generalized procedure. The difference 


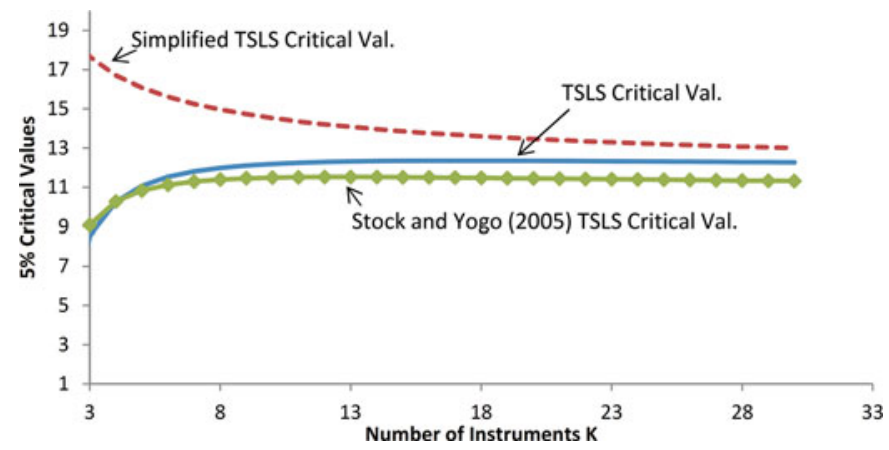

Figure 1. TSLS and simplified 5\% critical values assuming conditional homoscedasticity, no serial autocorrelation, and known $\boldsymbol{\Omega}$ and $\mathbf{W}=\boldsymbol{\Omega} \otimes I_{K}$. Under these assumptions, the effective number of degrees of freedom $K_{\text {eff }}$ equals the number of instruments $K$, and the effective $F$ statistic equals the nonrobust first-stage $F$ statistic. The null hypothesis is that the estimator Nagar bias exceeds $10 \%$ of the benchmark. Critical values are computed using the Patnaik (1949) methodology. For comparison, we show Stock and Yogo (2005) 5\% critical values of the null hypothesis that the asymptotic TSLS bias exceeds $10 \%$ of the OLS bias. The online version of this figure is in color.

between the simplified critical value and the TSLS and Stock and Yogo (2005) critical values decreases as $K$ becomes large.

\section{ASYMPTOTIC DISTRIBUTIONS AND AN EXAMPLE}

\subsection{Illustrative Example}

A simple example illustrates that heteroscedasticity and serial correlation impact the entire asymptotic distribution of both TSLS and LIML estimators, and can weaken the performance of the estimators. In this example, the first-stage $F$ statistic rejects the null hypothesis of weak instruments too often, while the effective $F$ statistic allows for testing for weak instruments with asymptotically correct size.

For the sake of exposition, assume $\beta=0$. Also assume that the departure from the conditionally homoscedastic serially uncorrelated framework takes the particularly simple form

$$
\mathbf{W}=a^{2}\left(\boldsymbol{\Omega} \otimes I_{K}\right),
$$

where $a$ is a scalar parameter and for $a=1$ the expression (6) reduces to the conditionally homoscedastic case.

Remark 1. We can generate example (6) with a purely conditionally heteroscedastic data-generating process. Let $\left\{\mathbf{Z}_{s}, \tilde{v}_{1 s}, \tilde{v}_{2 s}\right\}$ identically and independently distributed (iid). Let instruments independent with $\mathbb{E}\left[Z_{k s}\right]=0, \mathbb{E}\left[Z_{k s}^{2}\right]=1$, $\mathbb{E}\left[Z_{k s}^{3}\right]=0, \mathbb{E}\left[Z_{k s}^{4}\right]=a^{2}$. Let $\left(\tilde{v}_{1 s}, \tilde{v}_{2 s}\right) \sim N_{2}\left((0,0)^{\prime}, \boldsymbol{\Omega}\right)$ independently of $\mathbf{Z}_{s}$. Let the reduced form errors $v_{1 s}=$ $\tilde{v}_{1 s} \Pi_{k=1}^{K} Z_{k s}, v_{2 s}=\tilde{v}_{2 s} \Pi_{k=1}^{K} Z_{k s}$. Then $\mathbb{E}\left(\left[v_{1 s}, v_{2 s}\right]\left[v_{1 s}, v_{2 s}\right]^{\prime}\right)=$ $\boldsymbol{\Omega}$ and $\mathbb{E}\left(\left[v_{1 s}, v_{2 s}\right]\left[v_{1 s}, v_{2 s}\right]^{\prime} \otimes \mathbf{Z}_{s} \mathbf{Z}_{s}^{\prime}\right)=a^{2} \boldsymbol{\Omega} \otimes \mathbb{I}_{K}$. HL.1, HL.2, and (6) follow from the central limit theorem and the weak law of large numbers.

Remark 2. We can alternatively generate (6) with a simple serially correlated data-generating process. Assume that instruments and reduced form errors follow independent $\mathrm{AR}(1)$ processes $Z_{k s+1}=\rho_{Z} Z_{k s}+\epsilon_{k s+1}, k=$
$1, \ldots, K$, and $v_{j s+1}=\rho_{v} v_{j s}+\eta_{j s+1}, j=1,2$. Let $\epsilon_{k s}$ and $\eta_{j s}$ serially uncorrelated with mean zero, $\mathbb{E}\left(\boldsymbol{\epsilon}_{s} \boldsymbol{\epsilon}_{s}^{\prime}\right)=(1-$ $\left.\rho_{Z}^{2}\right) \times I_{K}$ and $\mathbb{E}\left[\eta_{1 s}, \eta_{2 s}\right]^{\prime}\left[\eta_{1 s}, \eta_{2 s}\right]=\left(1-\rho_{V}^{2}\right) \times \boldsymbol{\Omega}$. Then $\mathbb{E}\left[v_{1 s}, v_{2 s}\right]\left[v_{1 s}, v_{2 s}\right]^{\prime}=\boldsymbol{\Omega}$ and $\mathbb{E}\left(\mathbf{Z}_{s} \mathbf{Z}_{s}^{\prime}\right)=\mathbb{I}_{K}$. HL.1, HL.2 follow from the central limit theorem and the weak law of large numbers. Expression (6) holds with $a=\left(1+\rho_{v} \rho_{Z}\right) /(1-$ $\left.\rho_{Z} \rho_{v}\right)$. Serial correlation in both the instruments and the errors is required for $a \neq 1$. As a numerical example, moderate serial correlation of $\rho_{v}=\rho_{Z}=0.5$ gives rise to $a=1.67$.

With Assumptions $\mathrm{L}_{\Pi}$ and HL, the asymptotic distribution of the TSLS estimator

$$
\begin{aligned}
\widehat{\beta}_{\mathrm{TSLS}} \equiv & {\left[\mathbf{Y}^{\prime} \mathbf{Z}\left(\mathbf{Z}^{\prime} \mathbf{Z}\right)^{-1} \mathbf{Z}^{\prime} \mathbf{Y}\right]^{-1} \mathbf{Y}^{\prime} \mathbf{Z}\left(\mathbf{Z}^{\prime} \mathbf{Z}\right)^{-1} \mathbf{Z}^{\prime} \mathbf{v}_{1} } \\
= & \frac{\omega_{1}}{\omega_{2}}\left[\left(\frac{\mathbf{C}}{a \omega_{2}}+\frac{\mathbf{Z}^{\prime} \mathbf{v}_{2} / \sqrt{S}}{a \omega_{2}}\right)^{\prime}\left(\frac{\mathbf{C}}{a \omega_{2}}+\frac{\mathbf{Z}^{\prime} \mathbf{v}_{2} / \sqrt{S}}{a \omega_{2}}\right)\right]^{-1} \\
& \times\left(\frac{\mathbf{C}}{a \omega_{2}}+\frac{\mathbf{Z}^{\prime} \mathbf{v}_{2} / \sqrt{S}}{a \omega_{2}}\right)^{\prime} \frac{\mathbf{Z}^{\prime} \mathbf{v}_{1} / \sqrt{S}}{a \omega_{1}} \\
\stackrel{d}{\rightarrow} & \frac{\omega_{1}}{\omega_{2}}\left[\boldsymbol{\psi}_{2}^{\prime} \boldsymbol{\psi}_{2}\right]^{-1} \boldsymbol{\psi}_{2}^{\prime} \boldsymbol{\psi}_{1},
\end{aligned}
$$

where

$$
\begin{aligned}
& \left(\begin{array}{l}
\boldsymbol{\psi}_{1} \\
\boldsymbol{\psi}_{2}
\end{array}\right) \\
& \sim \mathcal{N}_{2 K}\left(\left(\begin{array}{c}
\mathbf{0}_{\mathbf{K}} \\
\mathbf{C} /\left(a \omega_{2}\right)
\end{array}\right),\left(\begin{array}{cc}
1 & \omega_{12} /\left(\omega_{1} \omega_{2}\right) \\
\omega_{12} /\left(\omega_{1} \omega_{2}\right) & 1
\end{array}\right) \otimes \mathbb{I}_{K}\right) .
\end{aligned}
$$

The asymptotic TSLS distribution depends only on the elements of the noncentral Wishart matrix $\left[\boldsymbol{\psi}_{1}, \boldsymbol{\psi}_{2}\right]^{\prime}\left[\boldsymbol{\psi}_{1}, \boldsymbol{\psi}_{2}\right]$. Hence, the vector of first-stage coefficients $\mathbf{C}$ and the parameter $a$ enter into the asymptotic distribution in (10) only through the noncentrality parameter $\mathbf{C}^{\prime} \mathbf{C} / a^{2} \omega_{2}^{2}$, so $\mathbf{C}^{\prime} \mathbf{C} / a^{2} \omega_{2}^{2}$ summarizes instrument strength.

In this example, heteroscedasticity and serial correlation affect the biases and test size distortion of TSLS and LIML estimators in the same way as a weaker first-stage relationship. The conditionally homoscedastic serially uncorrelated case obtains for $a=1$, so the TSLS estimator is asymptotically distributed as if the errors were conditionally homoscedastic serially uncorrelated, and the first-stage coefficients were reduced by a factor of $a$. We prove an analogous result for LIML in the Appendix.

Consider a null hypothesis for weak instruments of the form $\left(\mathbf{C}^{\prime} \mathbf{C} / \omega_{2}^{2} a^{2} K\right)<x$. In the presence of conditional heteroscedasticity or serial correlation of the form (6), the first-stage $F$ statistic is asymptotically distributed as $a^{2} \chi_{K}^{2}\left(\mathbf{C}^{\prime} \mathbf{C} / \omega_{2}^{2} a^{2}\right) / K$. As $a$ increases without bound, the noncentrality parameter goes to zero and instruments become arbitrarily weak, but the firststage $F$ statistic diverges to infinity almost surely. On the other hand, the effective $F$ statistic is asymptotically distributed as a $\chi_{K}^{2}\left(\mathbf{C}^{\prime} \mathbf{C} / \omega_{v}^{2} a^{2}\right) / K$, so we can reject the null hypothesis of weak instruments with confidence level $\alpha$ whenever $\widehat{F}_{\text {eff }}$ exceeds the upper $\alpha$ quantile of $\chi_{K}^{2}(x \times K) / K$. 


\subsection{Asymptotic Distributions}

Definition 1. Denote the projection matrix onto $\mathbf{Z}$ by $\mathbf{P}_{\mathbf{Z}}=$ $\mathbf{Z Z}^{\prime} / S$ and the complementary matrix by $\mathbf{M}_{\mathbf{Z}}=\mathbb{I}_{S}-\mathbf{P}_{\mathbf{Z}}$.

1. The TSLS estimator

$$
\widehat{\beta}_{\mathrm{TSLS}} \equiv\left(\mathbf{Y}^{\prime} \mathbf{P}_{\mathbf{Z}} \mathbf{Y}\right)^{-1}\left(\mathbf{Y}^{\prime} \mathbf{P}_{\mathbf{Z}} \mathbf{y}\right) .
$$

2. The limited information likelihood (LIML) estimator

$$
\widehat{\beta}_{\mathrm{LIML}}=\left(\mathbf{Y}^{\prime}\left(\mathbb{I}_{S}-k_{\mathrm{LIML}} \mathbf{M}_{\mathbf{Z}}\right) \mathbf{Y}\right)^{-1}\left(\mathbf{Y}^{\prime}\left(\mathbb{I}_{S}-k \mathbf{M}_{\mathbf{Z}}\right) \mathbf{y}\right),
$$

where $k_{\text {LIML }}$ is the smallest root of the determinantal equation

$$
\left|[\mathbf{y}, \mathbf{Y}]^{\prime}[\mathbf{y}, \mathbf{Y}]-k[\mathbf{y}, \mathbf{Y}]^{\prime} \mathbf{M}_{\mathbf{Z}}[\mathbf{y}, \mathbf{Y}]\right|=0 .
$$

3. The nonrobust first-stage F statistic

$$
\widehat{F} \equiv \frac{\mathbf{Y}^{\prime} \mathbf{P}_{\mathbf{Z}} \mathbf{Y}}{K \widehat{\omega}_{2}^{2}},
$$

where $\widehat{\omega}_{2}^{2} \equiv \frac{\left(\mathbf{Y}-\mathbf{P}_{\mathbf{Z}} \mathbf{Y}\right)^{\prime}\left(\mathbf{Y}-\mathbf{P}_{\mathbf{Z}} \mathbf{Y}\right)}{S-K-1}$.

4. The robust first-stage $F$ statistic

$$
\widehat{F}_{r} \equiv \frac{\mathbf{Y}^{\prime} \mathbf{Z} \widehat{\mathbf{W}}_{2}^{-1} \mathbf{Z}^{\prime} \mathbf{Y}}{K \times S},
$$

where $\widehat{\mathbf{W}}_{2}$ is the lower diagonal $K \times K$ block of the matrix $\widehat{\mathbf{W}}$.

5. The effective first-stage $F$ statistic

$$
\widehat{F}_{\text {eff }} \equiv \frac{\mathbf{Y}^{\prime} \mathbf{P}_{\mathbf{Z}} \mathbf{Y}}{\operatorname{tr}\left(\widehat{\mathbf{W}}_{2}\right)}
$$

Lemma 1 derives asymptotic distributions for these statistics, generalizing Theorem 1 in Staiger and Stock (1997).

Lemma 1. Write $\sigma_{1}^{2}=\omega_{1}^{2}-2 \beta \omega_{12}+\beta^{2} \omega_{2}^{2}, \sigma_{12}=\omega_{12}-$ $\beta \omega_{2}^{2}, \sigma_{2}^{2}=\omega_{2}^{2}$, and $\boldsymbol{\Sigma}=\left(\begin{array}{cc}\sigma_{1}^{2} & \sigma_{12} \\ \sigma_{12} & \sigma_{2}^{2}\end{array}\right)$. Under Assumptions $\mathrm{L}_{\Pi}$ and HL, the following limits hold jointly as $S \rightarrow \infty$.

1. $\widehat{\beta}_{\mathrm{TSLS}}-\beta \stackrel{d}{\rightarrow} \beta_{\mathrm{TSLS}}^{*}=\left(\boldsymbol{\gamma}_{2}{ }^{\prime} \boldsymbol{\gamma}_{2}\right)^{-1} \boldsymbol{\gamma}_{2}{ }^{\prime}\left(\boldsymbol{\gamma}_{1}-\beta \boldsymbol{\gamma}_{2}\right)$.

2. $\widehat{\beta}_{\mathrm{LIML}}-\beta \stackrel{d}{\rightarrow} \beta_{\mathrm{LIML}}^{*}=\left(\boldsymbol{\gamma}_{2}^{\prime} \boldsymbol{\gamma}_{2}-\kappa_{\mathrm{LIML}} \omega_{2}^{2}\right)^{-1}\left(\boldsymbol{\gamma}_{2}^{\prime}\left(\boldsymbol{\gamma}_{1}-\right.\right.$ $\left.\left.\beta \gamma_{2}\right)-\kappa_{\text {LIML }}\left(\omega_{12}-\beta \omega_{2}^{2}\right)\right)$,

where $\kappa_{\text {LIML }}$ is the smallest root of $\mid\left[\boldsymbol{\gamma}_{1}-\beta \boldsymbol{\gamma}_{2}, \boldsymbol{\gamma}_{2}\right]^{\prime}\left[\boldsymbol{\gamma}_{1}-\right.$ $\left.\beta \gamma_{2}, \gamma_{2}\right]-\kappa \boldsymbol{\Sigma} \mid=0$.

3. $\widehat{F} \stackrel{d}{\rightarrow} F^{*} \equiv \boldsymbol{\gamma}_{2}{ }^{\prime} \boldsymbol{\gamma}_{2} / K \omega_{2}^{2}$.

4. $\widehat{F}_{r} \stackrel{d}{\rightarrow} F_{r}^{*} \equiv \boldsymbol{\gamma}_{2}^{\prime} \mathbf{W}_{2}^{-1} \boldsymbol{\gamma}_{2} / K$.

5. $\widehat{F}_{\text {eff }} \stackrel{d}{\rightarrow} F_{\text {eff }}^{*} \equiv \gamma_{2}{ }^{\prime} \gamma_{2} / \operatorname{tr}\left(\mathbf{W}_{2}\right)$,

where

$$
\left(\begin{array}{l}
\boldsymbol{\gamma}_{1} \\
\boldsymbol{\gamma}_{2}
\end{array}\right) \sim \mathcal{N}_{2 K}\left(\left(\begin{array}{c}
\beta \mathbf{C} \\
\mathbf{C}
\end{array}\right), \mathbf{W}\right)
$$

Proof. See the Appendix.

The limiting distributions are functions of a multivariate normal vector whose distribution depends on the parameters $(\beta, \mathbf{C})$, and on the matrix W. We treat the asymptotic distributions in Lemma 1 as a limiting experiment in the sense of Müller (2011), and use it to analyze inference problems regarding $(\beta, \mathbf{C})$.

\section{TESTING THE NULL HYPOTHESIS OF WEAK INSTRUMENTS}

We base our null hypothesis of weak instruments on a bias criterion. We follow the standard methodology in Nagar (1959), and approximate the asymptotic TSLS and LIML distributions to obtain the Nagar bias. Under standard asymptotics, the Nagar bias for both estimators is zero everywhere in the parameter space, but under weak instrument asymptotics, the bias may be large in some regions of the parameter space. We consider instruments to be weak when the estimator Nagar bias is large relative to a benchmark, extending the OLS bias criterion in Stock and Yogo (2005).

\subsection{Nagar Approximation}

Theorem 1. (Nagar Approximation) Let $\mathbf{W} \in \mathbb{R}^{2 K \times 2 K}$ positive definite. Write $\mathbf{C} \in \mathbb{R}^{K}$ as $\mathbf{C}=\|\mathbf{C}\| \mathbf{C}_{0}$ and let $\mu^{2} \equiv$ $\|\mathbf{C}\|^{2} / \operatorname{tr}\left(\mathbf{W}_{2}\right)$. Define $\quad \mathbf{S}_{1}=\mathbf{W}_{1}-2 \beta \mathbf{W}_{12}+\beta^{2} \mathbf{W}_{2}, \quad \mathbf{S}_{12}=$ $\mathbf{W}_{12}-\beta \mathbf{W}_{2}, \mathbf{S}_{2}=\mathbf{W}_{2}$, and the benchmark $\operatorname{BM}(\beta, \mathbf{W}) \equiv$ $\sqrt{\operatorname{tr}\left(\mathbf{S}_{1}\right) / \operatorname{tr}\left(\mathbf{S}_{2}\right)}$. We write $\mathcal{S}^{K-1}$ for the $K-1$ dimensional unit sphere.

1. For $e \in\{$ TSLS, LIML $\}$, the Taylor expansion of $\beta_{e}^{*}$ around $\mu^{-1}=0$ gives the Nagar (1959) bias

$$
N_{e}(\beta, \mathbf{C}, \mathbf{W}, \boldsymbol{\Omega})=\mu^{-2} n_{e}\left(\beta, \mathbf{C}_{0}, \mathbf{W}, \boldsymbol{\Omega}\right),
$$

with

$$
\begin{aligned}
& n_{\mathrm{TSLS}}\left(\beta, \mathbf{C}_{0}, \mathbf{W}, \boldsymbol{\Omega}\right)=\frac{\operatorname{tr}\left(\mathbf{S}_{12}\right)}{\operatorname{tr}\left(\mathbf{S}_{2}\right)}\left[1-2 \frac{\mathbf{C}_{0}^{\prime} \mathbf{S}_{12} \mathbf{C}_{0}}{\operatorname{tr}\left(\mathbf{S}_{12}\right)}\right], \\
& n_{\mathrm{LIML}}\left(\beta, \mathbf{C}_{0}, \mathbf{W}, \boldsymbol{\Omega}\right) \\
& \quad=\frac{\operatorname{tr}\left(\mathbf{S}_{12}\right)-\frac{\sigma_{12}}{\sigma_{1}^{2}} \operatorname{tr}\left(\mathbf{S}_{1}\right)-\mathbf{C}_{0}^{\prime}\left(2 \mathbf{S}_{12}-\frac{\sigma_{12}}{\sigma_{1}^{2}} \mathbf{S}_{1}\right) \mathbf{C}_{0}}{\operatorname{tr}\left(\mathbf{S}_{2}\right)},
\end{aligned}
$$

2. For $e \in\{$ TSLS, LIML $\}$ :

$$
B_{e}(\mathbf{W}, \boldsymbol{\Omega}) \equiv \sup _{\beta \in \mathbb{R}, \mathbf{C}_{0} \in \mathcal{S}^{K-1}}\left(\frac{\left|n_{e}\left(\beta, \mathbf{C}_{0}, \mathbf{W}, \boldsymbol{\Omega}\right)\right|}{\operatorname{BM}(\beta, \mathbf{W})}\right)<\infty .
$$

3. $B_{\mathrm{TSLS}}(\mathbf{W}, \boldsymbol{\Omega}) \leq 1$.

Proof. See the Appendix.

Remark 3. The Nagar bias is the bias of an approximating distribution. It equals the expectation of the first three terms in the Taylor series expansion of the asymptotic estimator distribution under weak instrument asymptotics. It is therefore always defined and bounded for both TSLS and LIML. While the asymptotic estimator bias may not always exist, our test is still performing well. Under the null hypothesis, the Nagar bias can be large, but under the alternative hypothesis, the Nagar bias is small; see Section 4.2. Under certain conditions, we can also prove that the Nagar bias approximates the asymptotic estimator bias as the concentration parameter $\mu^{2}$ goes to infinity; see supplementary materials C.1.

Remark 4. We interpret the benchmark $\operatorname{BM}(\beta, \mathbf{W})=$ $\sqrt{\operatorname{tr}\left(\mathbf{S}_{1}\right) / \operatorname{tr}\left(\mathbf{S}_{2}\right)}$ as a "worst-case" bias. An ad hoc approximation of $\mathbb{E}\left[\beta_{\mathrm{TSLS}}^{*}\right]$ as a ratio of expectations as in Staiger and 
Stock (1997) helps convey the intuition:

$$
\begin{aligned}
\mathbb{E}\left[\beta_{\mathrm{TSLS}}^{*}\right] & \approx \frac{\operatorname{tr}\left(\mathbf{S}_{12}\right)}{\operatorname{tr}\left(\mathbf{S}_{2}\right)\left[1+\mu^{2}\right]} \\
& \approx \frac{1}{\left[1+\mu^{2}\right]} \frac{\operatorname{tr}\left(\mathbf{S}_{12}\right)}{\sqrt{\operatorname{tr}\left(\mathbf{S}_{2}\right)} \sqrt{\operatorname{tr}\left(\mathbf{S}_{1}\right)}} \sqrt{\frac{\operatorname{tr}\left(\mathbf{S}_{1}\right)}{\operatorname{tr}\left(\mathbf{S}_{2}\right)}} .
\end{aligned}
$$

The first factor is maximized when instruments are completely uninformative and $\mu^{2}=0$, while the second factor is maximized when first- and second-stage errors are perfectly correlated (Liu and Neudecker 1995).

Remark 5. In the implementation of our generalized testing procedure, we use the function $B_{e}(\mathbf{W}, \boldsymbol{\Omega})$ to bound the Nagar bias relative to the benchmark. We provide a fast and accurate numerical MATLAB routine for $B_{e}(\mathbf{W}, \boldsymbol{\Omega})$. For any given value of the structural parameter $\beta$, we compute the supremum over $\mathbf{C}_{0} \in \mathcal{S}^{K-1}$ analytically using matrix diagonalization. We then compute the limits of $\sup _{\mathbf{C}_{0} \in \mathcal{S}^{K-1}}\left|n_{e}\left(\beta, \mathbf{C}_{0}, \mathbf{W}, \boldsymbol{\Omega}\right)\right| / \mathrm{BM}(\beta, \mathbf{W})$ as $\beta \rightarrow \pm \infty$. Finally, we numerically maximize the function $\sup _{\mathbf{C}_{0} \in \mathcal{S}^{K-1}}\left|n_{e}\left(\beta, \mathbf{C}_{0}, \widehat{\mathbf{W}}, \widehat{\boldsymbol{\Omega}}\right)\right| / \operatorname{BM}(\beta, \boldsymbol{\Omega}) \quad$ over $\beta \in[-X, X]$, where $X \in \mathbb{R}^{+}$is chosen sufficiently large.

\subsection{Null Hypothesis}

For a given threshold $\tau \in[0,1]$ and matrix $\mathbf{W} \in \mathbb{R}^{2 K \times 2 K}$, we define the null and alternative hypotheses for $e \in\{$ TSLS, LIML\}

$$
H_{e}^{0}: \mu^{2} \in \mathcal{H}_{e}(\mathbf{W}, \boldsymbol{\Omega}) \quad \text { versus } \quad H_{e}^{1}: \mu^{2} \notin \mathcal{H}_{e}(\mathbf{W}, \boldsymbol{\Omega}),
$$

where

$$
\begin{aligned}
\mathcal{H}_{e}(\mathbf{W}, \boldsymbol{\Omega}) \equiv & \left\{\mu^{2} \in \mathbb{R}_{+}: \sup _{\beta \in \mathbb{R}, C_{0} \in \mathcal{S}^{K-1}}\right. \\
& \left.\frac{\left|N_{e}\left(\beta, \mu \sqrt{\operatorname{tr} M \mathbf{W}_{2}} \mathbf{C}_{0}, \mathbf{W}, \boldsymbol{\Omega}\right)\right|}{\operatorname{BM}(\beta, \mathbf{W})}>\tau\right\} .
\end{aligned}
$$

Under the null hypothesis, the Nagar bias exceeds a fraction $\tau$ of the benchmark for at least some value of the structural parameter $\beta$ and some direction of the first-stage coefficients $\mathbf{C}_{0}$. On the other hand, under the alternative, the Nagar bias is at most a fraction $\tau$ of the benchmark for any values $\left(\beta, \mathbf{C}_{0}\right)$.

\subsection{Testing Procedures}

We base our test on the statistic $\widehat{F}_{\text {eff }}$, which is asymptotically distributed as a quadratic form in normal random variables with mean $1+\mu^{2}$; see Lemma 1 . For a survey of this class of distributions, see Johnson, Kotz, and Balakrishnan (1995, chap. 29). Denote by $F_{\mathbf{C}, \mathbf{W}_{2}}^{-1}(\alpha)$, the upper $\alpha$ quantile of the distribution $\boldsymbol{\gamma}_{2}^{\prime} \boldsymbol{\gamma}_{2} / \operatorname{tr}\left(\mathbf{W}_{2}\right)$, where $\boldsymbol{\gamma}_{2} \sim \mathcal{N}_{K}\left(\mathbf{C}, \mathbf{W}_{2}\right)$ and let

$$
c\left(\alpha, \mathbf{W}_{2}, x\right) \equiv \sup _{\mathbf{C} \in \mathbb{R}^{K}}\left\{F_{\mathbf{C}, \mathbf{W}_{2}}^{-1}(\alpha) \mathbb{1}_{\mathbf{C}^{\prime} \mathbf{C} / \operatorname{tr}\left(\mathbf{W}_{2}\right)<x}\right\}
$$

$\mathbb{1}_{A}(\cdot)$ denotes the indicator function over a set $\mathrm{A}$. We base the generalized test on the observation that $\mathcal{H}_{e}(\mathbf{W}, \boldsymbol{\Omega})=$ $\left[0, B_{e}(\mathbf{W}, \boldsymbol{\Omega}) / \tau\right)$. The generalized procedure is applicable to both TSLS and LIML, and it rejects the null hypothesis $H_{e}^{0}$ whenever

$$
\widehat{F}_{\text {eff }}>c\left(\alpha, \widehat{\mathbf{W}}_{2}, B_{e}(\widehat{\mathbf{W}}, \widehat{\boldsymbol{\Omega}}) / \tau\right) .
$$

Lemma 2. Under Assumptions $\mathrm{L}_{\Pi}$ and $\mathrm{HL}$, the generalized procedure is pointwise asymptotically valid, that is

$$
\sup _{\mathcal{H}_{e}(\mathbf{W}, \boldsymbol{\Omega})} \lim _{S \rightarrow \infty} \mathbb{P}\left(\widehat{F}_{\text {eff }}>c\left(\alpha, \widehat{\mathbf{W}}_{2}, B_{e}(\widehat{\mathbf{W}}, \widehat{\boldsymbol{\Omega}}) / \tau\right)\right) \leq \alpha .
$$

Furthermore, provided that $B(\widehat{\mathbf{W}}, \widehat{\boldsymbol{\Omega}})$ is bounded in probability

$$
\lim _{\mu^{2} \rightarrow \infty} \lim _{S \rightarrow \infty} \mathbb{P}\left(\widehat{F}_{\text {eff }}>c\left(\alpha, \widehat{\mathbf{W}}_{2}, B_{e}(\widehat{\mathbf{W}}, \widehat{\boldsymbol{\Omega}}) / \tau\right)=1 .\right.
$$

Proof. See the Appendix.

The inequality in Theorem 1.3 implies a simplified asymptotically valid test for TSLS, which rejects the null hypothesis $\mathcal{H}_{e}(\mathbf{W}, \mathbf{\Omega})$ whenever

$$
\widehat{F}_{\text {eff }}>c\left(\alpha, \widehat{\mathbf{W}}_{2}, 1 / \tau\right) .
$$

With $c\left(\alpha, \widehat{\mathbf{W}}_{2}, 1 / \tau\right) \geq c\left(\alpha, \widehat{\mathbf{W}}_{2}, B_{\mathrm{TSLS}}(\mathbf{W}, \boldsymbol{\Omega}) / \tau\right)$, the simplified procedure is asymptotically valid and weakly less powerful than the generalized procedure. The simplified test is conservative, in the sense that under the alternative hypothesis, the TSLS Nagar bias is lower than the threshold for any degree of dependence in the second stage.

\section{COMPUTATION OF CRITICAL VALUES}

We provide two simple methods to compute the critical value $c\left(\alpha, \mathbf{W}_{2}, x\right)$. Our first method generates Monte Carlo critical values $c_{m}\left(\alpha, \mathbf{W}_{2}, x\right)$. We obtain estimates of $F_{\mathbf{C}, \mathbf{W}_{2}}^{-1}(\alpha)$ as the sample upper $\alpha$ point from a large number of draws from the distribution of $\boldsymbol{\gamma}_{2}^{\prime} \boldsymbol{\gamma}_{2} / \operatorname{tr}\left(\widehat{\mathbf{W}}_{2}\right)$, and then maximize over a large set of $\mathbf{C}$, such that $\mathbf{C}^{\prime} \mathbf{C} / \operatorname{tr}\left(\mathbf{W}_{2}\right) \leq x$.

The second procedure is based on a curve-fitting methodology first suggested by Patnaik (1949). Patnaik (1949) and Imhof (1961) approximated the critical values of a weighted sum of independent noncentral chi-squared distributions by a central $\chi^{2}$ with the same first and second moments. We analogously approximate the distribution $F_{\mathbf{C}, \mathbf{W}_{2}}$ by a noncentral $\chi^{2}$ with the same first and second moments. Our approximation errors are therefore bounded by the original Patnaik errors through a triangle inequality. We use

$$
F_{\mathbf{C}, \mathbf{W}_{2}}^{-1}(\alpha) \approx \frac{1}{K_{\mathrm{eff}}} F_{\chi_{K_{\mathrm{eff}}}^{2}\left(K_{\mathrm{eff}} \mu^{2}\right)}^{-1}(\alpha),
$$

where $K_{\text {eff }}$ is possibly fractional with

$$
K_{\mathrm{eff}}=\left[\operatorname{tr}\left(\mathbf{W}_{2}\right)\right]^{2} \frac{1+2 \mu^{2}}{\operatorname{tr}\left(\mathbf{W}_{2}^{2}\right)+2 \mathbf{C}^{\prime} \mathbf{W}_{2} \mathbf{C}} .
$$

There is a large literature that approximates distributions by choosing a family of distributions and selecting the member that fits best, often by matching lower order moments of the original distribution (Satterthwaite 1946; Pearson 1959; Theil and Nagar 1961; Grubbs 1964; Henshaw 1966; Conerly and Mansfield 1988; Liu, Tang, and Zhang 2009). The noncentral chi-squared distribution is a natural choice, because it is exact in the homoscedastic case.

While it is hard to assess the accuracy of these curve-fitting approximations analytically, they are often simple and numerically highly accurate (Rothenberg 1984). We demonstrate the 
degree of accuracy of their approximations using numerical examples. In the supplementary materials B.1, we verify that the approximation (30) is numerically as accurate as the original central Patnaik distribution for the quadratic forms considered in Imhof (1961); approximation errors are at most $0.7 \%$ points in the important upper $15 \%$ tail of the distributions.

Numerical results, such as in Table 1, clearly indicate that upper $\alpha$ quantiles of (30) are decreasing in $K_{\text {eff }}$. Moreover, the upper $\alpha$ quantile in (30) is nondecreasing in the noncentrality parameter $\mu^{2}$ (Ghosh 1973). Taking the supremum over $\mathbf{C}$ with $\mathbf{C}^{\prime} \mathbf{C} / \operatorname{tr}\left(\mathbf{W}_{2}\right)<x$ suggests the Patnaik critical value.

Definition 2. (Patnaik Critical Value) Define the Patnaik critical value as

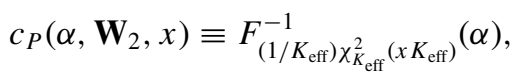

with the effective number of degrees of freedom

$$
K_{\text {eff }} \equiv \frac{\operatorname{tr}\left(\mathbf{W}_{2}\right)^{2}(1+2 x)}{\operatorname{tr}\left(\mathbf{W}_{2}^{2}\right)+2 \operatorname{tr}\left(\mathbf{W}_{2}\right) \max \operatorname{eval}\left(\mathbf{W}_{2}\right) x} .
$$

We numerically analyze the sizes of Monte Carlo and Patnaik critical values for benchmark parameter values $\alpha=5 \%$ and $x=10$, and find that size distortions are small for both methodologies. Monte Carlo critical values are computed with 40,000 draws from $\boldsymbol{\gamma}_{2}^{\prime} \boldsymbol{\gamma}_{2} / \operatorname{tr}\left(\mathbf{W}_{2}\right)$, and we replace the infinite set of vectors $\mathbf{C}$ s.t. $\mathbf{C}^{\prime} \mathbf{C} / \operatorname{tr}\left(\mathbf{W}_{2}\right)<x$ by a finite set of size 500 . We use code for $F_{\mathbf{C}, \mathbf{W}_{2}}(x)$ available from Ruud (2000) (Imhof 1961; Koerts and Abrahamse 1969; Farebrother 1990; Ruud 2000). For 400 matrices $\mathbf{W}_{2}$ from a diffuse prior with $K \in\{1,2,3,4,5\}$, our numerical values for $\max _{\mathbf{C}^{\prime} \mathbf{C} / \operatorname{trW}_{2}<x} F_{\mathbf{C}, \mathbf{W}_{2}}\left(c_{m}\right)$ range between $4.77 \%$ and $5.26 \%$, and

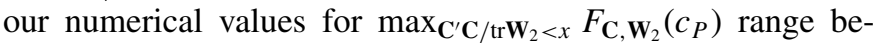
tween $5.00 \%$ and $5.02 \%$. For further details and MATLAB routines, see supplementary materials B.2-B.5.

Our generalized and simplified critical values differ from those proposed by Stock and Yogo (2005) for the TSLS bias, even when first- and second-stage errors are perfectly conditionally homoscedastic and serially uncorrelated. In this case, the effective $F$ statistic coincides with the Stock and Yogo (2005) test statistic. We obtain different critical values because, unlike them, we use an approximation to evaluate the weak instrument TSLS bias. Moreover, estimating $\widehat{\mathbf{W}}$ and $\widehat{\boldsymbol{\Omega}}$ also generates differences in critical values. The difference between our generalized TSLS critical values and analogous Stock and Yogo (2005) critical values becomes small as the number of instruments becomes large.

In the supplementary materials B.6, we tabulate Stock and Yogo (2005) 5\% critical values for testing the null hypothesis that the TSLS bias exceeds $10 \%$ of the OLS bias and our generalized and simplified critical values with a threshold of $10 \%$ and size $5 \%$, assuming conditional homoscedasticity and no serial correlation. TSLS critical values are smaller than Stock and Yogo (2005) critical values for $K=3,4$, but larger than Stock and Yogo (2005) critical values for $K \geq 5$. The difference between the TSLS and Stock and Yogo (2005) critical values is always less than 1. The LIML critical values decline more rapidly with the number of instruments than either the TSLS or simplified critical values. The simplified critical values exceed the generalized TSLS critical values, because they use a bound that applies for any form of the matrix $\mathbf{W}$.

\section{EMPIRICAL APPLICATION: ESTIMATING THE ELASTICITY OF INTERTEMPORAL SUBSTITUTION}

We now apply our pretesting procedure to an empirical example, and show that allowing for heteroscedasticity and time series correlation can affect pretesting conclusions.

The literature has focused on estimating the linearized Euler equation in two standard IV frameworks (Hansen and Singleton 1983; Hall 1988; Campbell and Mankiw 1989; Campbell 2003):

$$
\begin{aligned}
\Delta c_{t+1} & =v+\psi r_{t+1}+u_{t+1} \text { and } \mathbb{E}\left[\mathbf{Z}_{t-1} u_{t+1}\right]=0 \\
r_{t+1} & =\xi+(1 / \psi) \Delta c_{t+1}+\eta_{t+1} \text { and } \mathbb{E}\left[\mathbf{Z}_{t-1} \eta_{t+t}\right]=0,
\end{aligned}
$$

where $\psi$ is the EIS, $\Delta c_{t+1}$ is consumption growth at time $t+1$, $r_{t+1}$ is a real asset return, and $v$ is a constant. The vector of instruments is denoted by $\mathbf{Z}_{t-1}$. We follow the preferred choice of variables in Yogo (2004), using as $r_{t}$ the real return on the short-term interest rate, and as instruments the nominal interest rate, inflation, consumption growth, and the log dividend-price ratio, all lagged twice. We use quarterly data from Yogo (2004).

The EIS determines an agent's willingness to substitute consumption over time. Its magnitude is important for understanding the dynamics of consumption and asset returns (Epstein and Zin 1989, 1991; Campbell 2003). While time-varying volatility can introduce additional bias into the estimation of the EIS (Bansal and Yaron 2004), Yogo (2004) argued that under certain types of conditional heteroscedasticity the EIS can still be identified.

Table 2 compares pretests for weak instruments for 11 countries. Panel A shows weak instrument pretests with the ex-post real interest rate as the endogenous variable, while Panel B shows weak instrument pretests with consumption growth as the endogenous variable. The nonrobust first-stage $F$ statistic in column 1 is shown in bold whenever it exceeds the Stock and Yogo (2005) critical value 10.27. This is the 5\% critical value for testing the null hypothesis that the TSLS bias exceeds $10 \%$ of the OLS bias under the assumption of conditional homoscedasticity and no serial correlation. As in Yogo (2004), this homoscedastic pretest indicates strong instruments in Panel A, but cannot reject weak instruments in Panel B for almost all countries in the sample.

The second and third columns report the HAC robust firststage $F$ statistic and the effective $F$ statistic computed with a Newey-West kernel and six lags. We show 5\% critical values for TSLS, LIML, and simplified pretests for the null hypothesis that the respective Nagar bias exceeds $10 \%$ of the "worst-case" benchmark.

In panel $\mathrm{A}$, we see that allowing for heteroscedasticity and serial correlation changes the pretesting results for some countries, while for other countries all pretests yield the same conclusion. The effective $F$ statistic can be smaller or larger than the regular or robust $F$ statistics. Simplified critical values always exceed TSLS critical values. LIML critical values tend to be smallest.

The results in Table 2(A) for the United States are particularly striking. While the US regular $F$ statistic clearly exceeds 
Table 2. Estimating the Elasticity of Intertemporal Substitution: Weak Instrument Pretests

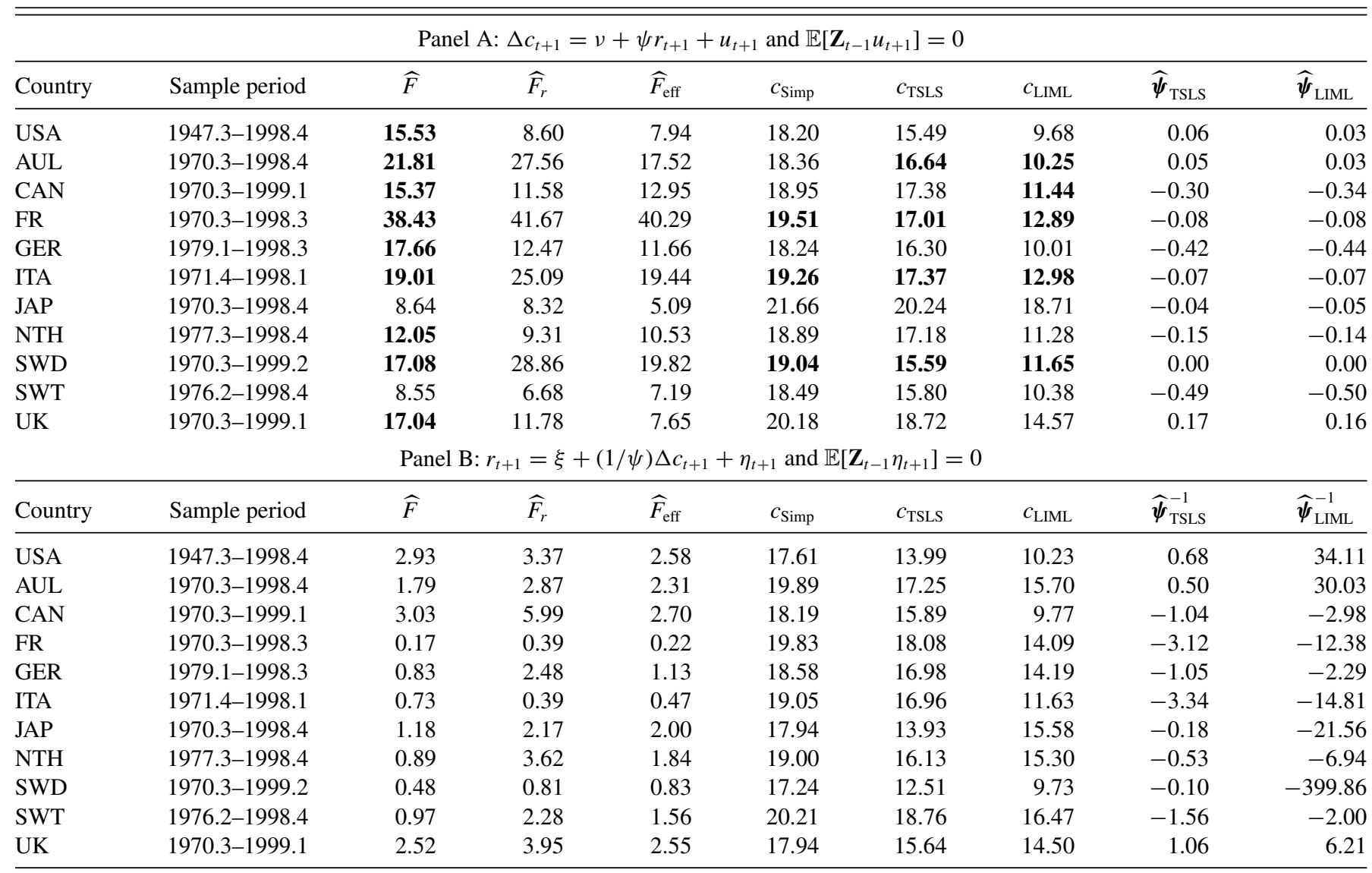

NOTE: $\Delta c$ is consumption growth and $r$ is the ex-post real short-term interest rate. We instrument using twice lagged nominal interest rate, inflation, dividend-price ratio, and consumption growth. HAC variance-covariance matrix $\widehat{\mathbf{W}}$ estimated with OLS and Newey-West kernel with six lags. $F$ statistic in bold when it exceeds the critical value of 10.27 . This is the 5\% critical value for testing the null hypothesis that the TSLS bias exceeds $10 \%$ of the OLS bias under the assumption of conditional homoscedasticity and no serial correlation (Stock and Yogo 2005). We show simplified, TSLS, and LIML critical values $c_{\mathrm{Simp}}=c_{P}\left(5 \%, \widehat{\mathbf{W}}_{2}, 10\right), c_{\mathrm{TSLS}}=c_{P}\left(5 \%, \widehat{\mathbf{W}}_{2}, 10 \times B_{\mathrm{TSLS}}(\widehat{\mathbf{W}}, \widehat{\boldsymbol{\Omega}})\right)$, and $c_{\mathrm{LIML}}=c_{P}\left(5 \%, \widehat{\mathbf{W}} 2,10 \times B_{\mathrm{LIML}}\left(\widehat{\mathbf{W}}_{2}, \widehat{\boldsymbol{\Omega}}\right)\right.$ ). Critical values are in bold when exceeded by $\widehat{F}_{\text {eff }} . \hat{\psi}_{\text {TSLS }}, \hat{\psi}_{\text {LIML }}, \widehat{(1 / \psi)}_{\text {TSLS }}$, and $\widehat{(1 / \psi)}_{\text {LIML }}$ are TSLS and LIML estimates of the EIS and its inverse.

the homoscedastic threshold of 10.27 , the robust and effective $F$ statistics are significantly smaller. The effective $F$ does not exceed the simplified, TSLS, or LIML critical values, so we cannot reject the null hypothesis of weak instruments under heteroscedasticity and serial correlation.

Panel B shows weak instrument pretests for the IV estimation of the inverse of the EIS. For this estimation, the results are consistent between homoscedastic and HAC weak instrument pretests. We cannot reject that instruments are weak for any of the countries in the sample.

The last two columns in Table 2 show the point estimates for $\psi$ and $1 / \psi$. For those cases where we can reject weak instruments under heteroscedasticity and serial correlation, the corresponding EIS point estimates are close to zero and often negative. Additional caution is, however, warranted in this interpretation, because as the number of countries increases, we are more and more likely to reject weak instruments at least once.

Our results confirm Yogo's (2004) finding that the EIS is small and close to zero. However, we also note that conditional heteroscedasticity and serial correlation may further weaken instruments and may affect TSLS and LIML bias in several of the country-specific regressions.

\section{CONCLUSION}

Heteroscedasticity, serial correlation, and panel data clustering can affect instrument strength. This article develops a robust test for weak instruments, which allows empirical researchers to test the null hypothesis that the TSLS or LIML Nagar bias is large relative to a benchmark.

The test is based on a scaled version of the regular $F$ statistic. Critical values depend on the covariance matrix of the reduced form coefficients and errors. Our general test requires computational work to evaluate the Nagar bias of TSLS or LIML. A simplified conservative version does not require this step, but is only available for TSLS. Critical values can then be implemented as quantiles of a noncentral chi-squared distribution with noninteger degrees of freedom.

Pretests based on the robust (or nonrobust) first-stage $F$ statistic with Stock and Yogo (2005) critical values are commonly applied outside the conditionally homoscedastic serially uncorrelated framework. However, to the best of our knowledge, there is no analysis supporting this practice. This article offers an alternative: a simple, asymptotically valid test that should be used for conditionally heteroscedastic, time series, and clustered panel data. 


\section{APPENDIX}

\section{A.1 Proof of Lemma 1}

First note the preliminary result that under Assumptions $L_{\Pi}$ and HL

$$
\begin{aligned}
\frac{1}{\sqrt{S}}\left(\begin{array}{c}
\mathbf{Z}^{\prime} \mathbf{y} \\
\mathbf{Z}^{\prime} \mathbf{Y}
\end{array}\right) & =\left(\begin{array}{c}
\beta \mathbf{C}+\mathbf{Z}^{\prime} \mathbf{v}_{1} / \sqrt{S} \\
\mathbf{C}+\mathbf{Z}^{\prime} \mathbf{v}_{2} / \sqrt{S}
\end{array}\right) \\
& \stackrel{d}{\rightarrow}\left(\begin{array}{l}
\boldsymbol{\gamma}_{1} \\
\boldsymbol{\gamma}_{2}
\end{array}\right) .
\end{aligned}
$$

1. $\widehat{\beta}_{\mathrm{TSLS}} \equiv\left(\mathbf{Y}^{\prime} \mathbf{P}_{\mathbf{Z}} \mathbf{Y}\right)^{-1}\left(\mathbf{Y}^{\prime} \mathbf{P}_{\mathbf{Z}} \mathbf{y}\right)=\left(\mathbf{Y}^{\prime} \mathbf{Z}\left(\mathbf{Z}^{\prime} \mathbf{Z}\right)^{-1} \mathbf{Z}^{\prime} \mathbf{Y}\right)^{-1}\left(\mathbf{Y}^{\prime} \mathbf{Z}\right.$ $\left.\left(\mathbf{Z}^{\prime} \mathbf{Z}\right)^{-1} \mathbf{Z}^{\prime} \mathbf{y}\right)$. Since we have assumed that $\mathbf{Z}^{\prime} \mathbf{Z} / S=\mathbb{I}_{K}$, the result follows from (A.2) and the continuous mapping theorem.

2. Write $\mathbf{J}=\left[\begin{array}{cc}1 & 0 \\ -\beta & 1\end{array}\right]$ and $\kappa=S(k-1)$. Note that $\mathbf{J}$ is nonsingular and so the roots of $\left|[\mathbf{y}, \mathbf{Y}]^{\prime}[\mathbf{y}, \mathbf{Y}]-k[\mathbf{y}, \mathbf{Y}]^{\prime} \mathbf{M}_{\mathbf{Z}}[\mathbf{y}, \mathbf{Y}]\right|=$ 0 are the same as of $\mid \mathbf{J}^{\prime}[\mathbf{y}, \mathbf{Y}]^{\prime}[\mathbf{y}, \mathbf{Y}] \mathbf{J}-k \mathbf{J}^{\prime}[\mathbf{y}, \mathbf{Y}]^{\prime} \mathbf{M}_{\mathbf{Z}}$ $[\mathbf{y}, \mathbf{Y}] \mathbf{J} \mid=0$. Moreover, $[\mathbf{y}, \mathbf{Y}]^{\prime}[\mathbf{y}, \mathbf{Y}]-(1+\kappa / S)[\mathbf{y}, \mathbf{Y}]^{\prime}$ $\mathbf{M}_{\mathbf{Z}}[\mathbf{y}, \mathbf{Y}]=[\mathbf{y}, \mathbf{Y}]^{\prime} \mathbf{P}_{\mathbf{Z}}[\mathbf{y}, \mathbf{Y}]-\kappa[\mathbf{y}, \mathbf{Y}]^{\prime} \mathbf{M}_{\mathbf{Z}}[\mathbf{y}, \mathbf{Y}] / S \stackrel{d}{\rightarrow}\left[\boldsymbol{\gamma}_{1}\right.$, $\left.\boldsymbol{\gamma}_{2}\right]^{\prime}\left[\boldsymbol{\gamma}_{1}, \boldsymbol{\gamma}_{2}\right]-\kappa \boldsymbol{\Omega}$ uniformly in $\kappa$ over compact sets. The solutions of $\left|[\mathbf{y}, \mathbf{Y}]^{\prime}[\mathbf{y}, \mathbf{Y}]-(1+\kappa / S)[\mathbf{y}, \mathbf{Y}]^{\prime} \mathbf{M}_{\mathbf{Z}}[\mathbf{y}, \mathbf{Y}]\right|=$ 0 , therefore, converge to those of $\mid \mathbf{J}^{\prime}\left[\boldsymbol{\gamma}_{1}, \boldsymbol{\gamma}_{2}\right]^{\prime}\left[\boldsymbol{\gamma}_{1}, \boldsymbol{\gamma}_{2}\right] \mathbf{J}-$ $\kappa \mathbf{J}^{\prime} \mathbf{\Omega} \mathbf{J} \mid=0$. With $\mathbf{J}^{\prime} \boldsymbol{\Omega} \mathbf{J}=\mathbf{\Sigma}$ thus $S\left(\hat{k}_{\text {LIML }}-1\right) \stackrel{d}{\rightarrow} \kappa_{\text {LIML }}$, where $\kappa_{\text {LIML }}$ is as given in Lemma 1.2.

Then $\widehat{\beta}_{\text {LIML }}-\beta=\left[\mathbf{Y}^{\prime}\left(\mathbb{I}_{S}-\hat{k}_{\text {LIML }} \mathbf{M}_{\mathbf{Z}}\right) \mathbf{Y}\right]^{-1}\left[\mathbf{Y}^{\prime}\left(\mathbb{I}_{S}-\hat{k}_{\mathrm{LIML}}\right.\right.$ $\left.\left.\mathbf{M}_{\mathbf{Z}}\right)(\mathbf{y}-\beta \mathbf{Y})\right]=\left[\mathbf{Y}^{\prime} \mathbf{P}_{\mathbf{Z}} \mathbf{Y}-S\left(\hat{k}_{\mathrm{LIML}}-1\right) \frac{\mathbf{Y}^{\prime} \mathbf{M}_{\mathbf{Z}} \mathbf{Y}}{S}\right]^{-1}\left[\mathbf{Y}^{\prime} \mathbf{P}_{\mathbf{Z}}\right.$ $\left.(\mathbf{y}-\beta \mathbf{Y})-S\left(\hat{k}_{\mathrm{LIML}}-1\right) \frac{\mathbf{Y}^{\prime} \mathbf{M}_{\mathbf{Z}}(\mathbf{y}-\beta \mathbf{Y})}{S}\right] \stackrel{d}{\rightarrow}\left[\boldsymbol{\gamma}_{2}^{\prime} \boldsymbol{\gamma}_{2}-\kappa_{\mathrm{LIML}}\right.$ $\left.\sigma_{2}^{2}\right]^{-1}\left[\boldsymbol{\gamma}_{2}\left(\boldsymbol{\gamma}_{1}-\beta \boldsymbol{\gamma}_{2}\right)-\kappa_{\text {LIML }} \sigma_{12}\right]$.

3. Note that $\widehat{\omega}_{2}^{2} \equiv\left(\mathbf{Y}-\mathbf{P}_{\mathbf{Z}} \mathbf{Y}\right)^{\prime}\left(\mathbf{Y}-\mathbf{P}_{\mathbf{Z}} \mathbf{Y}\right) /(S-K-1)=$ $\left(\mathbf{v}_{2}-\mathbf{P}_{\mathbf{Z}} \mathbf{v}_{2}\right)^{\prime}\left(\mathbf{v}_{2}-\mathbf{P}_{\mathbf{Z}} \mathbf{v}_{2}\right) /(S-K-1) \stackrel{d}{\rightarrow} \omega_{2}^{2}$ by Assumptions $\mathrm{L}_{\Pi}$ and HL. The result follows from (A.2) and the continuous mapping theorem.

4. and 5. follow from (A.2), the continuous mapping theorem, and Assumptions $\mathrm{L}_{\Pi}$ and $\mathrm{HL}$.

\section{A.2 LIML Distribution in Illustrative Example}

We show that in the illustrative example heteroscedasticity and serial correlation can effectively make instruments weaker for LIML. Assume $\mathbf{W}=a^{2} \boldsymbol{\Omega} \otimes \mathbb{I}_{K}$. Remember that $\hat{\beta}_{\mathrm{LIML}}=$ $\arg \min _{\tilde{\beta}}(\mathbf{y}-\tilde{\beta} Y)^{\prime} \mathbf{P}_{\mathbf{Z}}(\mathbf{y}-\tilde{\beta} \mathbf{Y}) /(\mathbf{y}-\tilde{\beta} \mathbf{Y})^{\prime}(\mathbf{y}-\tilde{\beta} \mathbf{Y})$. We will analyze the weak instrument limit of the LIML objective function. Note that, using Assumptions $\mathrm{L}_{\Pi}$ and $\mathrm{HL}, \mathbf{Z}^{\prime}(\mathbf{y}-$ $\tilde{\beta} \mathbf{Y}) / \sqrt{S} \stackrel{d}{\rightarrow} \boldsymbol{\gamma}_{1}-\tilde{\beta} \boldsymbol{\gamma}_{2}$.

Moreover, $(\mathbf{y}-\tilde{\beta} \mathbf{Y})^{\prime}(\mathbf{y}-\tilde{\beta} \mathbf{Y}) / S \stackrel{p}{\rightarrow} \omega_{1}^{2}-2 \tilde{\beta} \omega_{12}+\tilde{\beta}^{2} \omega_{2}^{2}$ uniformly in $\tilde{\beta}$ over compact sets. Hence, $\beta_{\text {LIML }}^{*}$ is distributed according to

$$
\arg \min _{\tilde{\beta}} a^{2} \frac{\left(\omega_{1} \boldsymbol{\psi}_{1}-\tilde{\beta} \omega_{2} \boldsymbol{\psi}_{2}\right)^{\prime}\left(\omega_{1} \boldsymbol{\psi}_{1}-\tilde{\beta} \omega_{2} \boldsymbol{\psi}_{2}\right)}{\omega_{1}^{2}-2 \tilde{\beta} \omega_{12}+\tilde{\beta}^{2} \omega_{2}^{2}} .
$$

Just as for the $\beta_{\mathrm{TSLS}}$, the vector of first-stage coefficients $\mathbf{C}$ and the parameter $a$ enter into the asymptotic distribution $\beta_{\text {LIML }}^{*}$ only through the noncentrality parameter $\mathbf{C}^{\prime} \mathbf{C} /\left(a^{2} \omega_{2}^{2}\right)$.

\section{A.3 Proof of Theorem 1}

A.3.1 Proof of Theorem 1.1. We follow Rothenberg (1984) in developing the Nagar (1959) moments for the TSLS and LIML estimators. We need to expand $\beta_{\mathrm{TSLS}}^{*}$ and $\beta_{\mathrm{LIML}}^{*}$ as second-order Taylor expansions in $\mu^{-1}$ around $\mu^{-1}=0$.

We start by developing the Taylor expansion for $\kappa_{\text {LIML }}$. Write $\mathbf{z}_{u}=\mathbf{S}_{1}^{-1 / 2}\left(\boldsymbol{\gamma}_{1}-\beta \boldsymbol{\gamma}_{2}\right)$ and $\mathbf{z}_{v}=\mathbf{S}_{2}^{-1 / 2}\left(\boldsymbol{\gamma}_{2}-C\right)$, so $\mathbf{z}_{u}$ and $\mathbf{z}_{v}$ are standard multivariate normal. Also write $\lambda=$ $\mu \operatorname{tr}\left(\mathbf{S}_{2}\right)^{1 / 2} \mathbf{S}_{2}^{-1 / 2} \mathbf{C}_{0}$, where $\mathbf{C}_{0}=\mathbf{C} /\|\mathbf{C}\|$.

$\kappa_{\text {LIML }}$ is defined as the smallest root of the determinantal equation

$$
\operatorname{det}\left(\mathbf{A}-\kappa_{\text {LIML }}\left[\begin{array}{cc}
\sigma_{1}^{2} & \sigma_{12} \\
\sigma_{12} & \sigma_{2}^{2}
\end{array}\right]\right)=0
$$

where

$$
\mathbf{A}=\left[\begin{array}{cc}
\mathbf{z}_{u}^{\prime} \mathbf{S}_{1} \mathbf{z}_{u} & \mathbf{z}_{u}^{\prime} \mathbf{S}_{1}^{1 / 2} \mathbf{S}_{2}^{1 / 2}\left(\lambda+\mathbf{z}_{v}\right) \\
\mathbf{z}_{u}^{\prime} \mathbf{S}_{1}^{1 / 2} \mathbf{S}_{2}^{1 / 2}\left(\lambda+\mathbf{z}_{v}\right) & \left(\mathbf{z}_{v}+\lambda\right)^{\prime} \mathbf{S}_{2}\left(\mathbf{z}_{v}+\lambda\right)
\end{array}\right] .
$$

We can rewrite this as a quadratic equation

$$
\begin{aligned}
& \left(\frac{\kappa_{\text {LIML }}}{\mu^{2}}\right)^{2}-\frac{\sigma_{v}^{2} a_{11}+\sigma_{1}^{2} a_{22}-2 a_{12} \sigma_{12}}{\mu^{2} \operatorname{det} \boldsymbol{\Sigma}} \frac{\kappa_{\text {LIML }}}{\mu^{2}} \\
& +\frac{\operatorname{det} \mathbf{A}}{\mu^{4} \operatorname{det} \boldsymbol{\Sigma}}=0 .
\end{aligned}
$$

We use the method of undetermined coefficients. Write

$$
\kappa_{\text {LIML }} \mu^{-2}=c_{0}+c_{1} \mu^{-1}+c_{2} \mu^{-2}+O\left(\mu^{-3}\right)
$$

for unknown constants $c_{0}, c_{1}, c_{2}$. Similarly write

$$
\begin{aligned}
d(\mu) & \equiv \frac{\sigma_{2}^{2} a_{11}+\sigma_{u}^{2} a_{22}-2 a_{12} \sigma_{12}}{\mu^{2} \operatorname{det} \mathbf{\Sigma}} \\
& =d_{0}+d_{1} \mu^{-1}+d_{2} \mu^{-2}+O\left(\mu^{-2}\right) \\
e(\mu) & \equiv \frac{\operatorname{det} \mathbf{A}}{\mu^{4} \operatorname{det} \mathbf{\Sigma}}=\frac{\operatorname{det} \mathbf{A}}{\mu^{4} \operatorname{det} \mathbf{\Sigma}}, \\
& =e_{0}+e_{1} \mu^{-1}+e_{2} \mu^{-2}+O\left(\mu^{-3}\right),
\end{aligned}
$$

where the Taylor series expansions for $d$ and $e$ give $d_{0}=\sigma_{1}^{2} \operatorname{tr}\left(\mathbf{S}_{2}\right) / \operatorname{det} \boldsymbol{\Sigma}, e_{0}=0, e_{1}=0$, and $e_{2}=\operatorname{tr}\left(\mathbf{S}_{2}\right)\left[\mathbf{z}_{u}^{\prime} \mathbf{S}_{1} \mathbf{z}_{u}-\right.$ $\left.\left(\mathbf{z}_{u}^{\prime} \mathbf{S}_{1}^{1 / 2} \mathbf{C}_{0}\right)^{2}\right] / \operatorname{det} \boldsymbol{\Sigma}$.

Substituting (A.5), (A.6), and (A.7) into the quadratic Equation (A.4) and equating coefficients gives $c_{0}\left(c_{0}-d_{0}\right)=0$. Since we are interested in the smaller solution, we have $c_{0}=0$. Then $c_{0}=0, c_{1}=0, c_{2}=\varepsilon_{2} / d_{0}$, and so $\kappa_{\text {LIML }} \mu^{-2}=\frac{1}{\sigma_{1}^{2}}\left[\mathbf{z}_{u}^{\prime} \mathbf{S}_{1} \mathbf{z}_{u}\right.$ $\left.\left(\mathbf{z}_{u}^{\prime} \mathbf{S}_{1}^{1 / 2} \mathbf{C}_{0}\right)^{2}\right] \mu^{-2}+O\left(\mu^{-3}\right)$.

We then expand $\beta_{\text {LIML }}^{*}$

$$
\begin{aligned}
\beta_{\text {LIML }}^{*}= & \mu^{-1} \frac{\mathbf{C}_{0}^{\prime} \mathbf{S}_{1}^{1 / 2} \mathbf{z}_{u} \operatorname{tr}\left(\mathbf{S}_{2}\right)^{1 / 2}}{\operatorname{tr}\left(\mathbf{S}_{2}\right)}+\mu^{-2} \frac{1}{\operatorname{tr}\left(\mathbf{S}_{2}\right)}\left(\mathbf{z}_{v}^{\prime} \mathbf{S}_{2}^{1 / 2} \mathbf{S}_{1}^{1 / 2} \mathbf{z}_{u}\right. \\
& \left.-2\left(\mathbf{C}_{0}^{\prime} \mathbf{S}_{1}^{1 / 2} \mathbf{z}_{u}\right)\left(\mathbf{C}_{0}^{\prime} \mathbf{S}_{2}^{1 / 2} \mathbf{z}_{v}\right)-c_{2} \sigma_{12}\right)+O\left(\mu^{-3}\right) .
\end{aligned}
$$

Taking the expectation of the first two terms gives the LIML Nagar bias as in the theorem. 
We can similarly derive the Taylor expansion for $\beta_{\mathrm{TSLS}}^{*}$ according to

$$
\begin{aligned}
\beta_{\mathrm{TSLS}}^{*}= & \mu^{-1} \frac{\mathbf{C}_{0}^{\prime} \mathbf{S}_{1}^{1 / 2} \mathbf{z}_{u} \operatorname{tr}\left(\mathbf{S}_{2}\right)^{1 / 2}}{\operatorname{tr}\left(\mathbf{S}_{2}\right)}+\mu^{-2} \frac{1}{\operatorname{tr}\left(\mathbf{S}_{2}\right)}\left(\mathbf{z}_{v}^{\prime} \mathbf{S}_{2}^{1 / 2} \mathbf{S}_{1}^{1 / 2} \mathbf{z}_{u}\right. \\
& \left.-2\left(\mathbf{C}_{0}^{\prime} \mathbf{S}_{1}^{1 / 2} \mathbf{z}_{u}\right)\left(\mathbf{C}_{0}^{\prime} \mathbf{S}_{2}^{1 / 2} \mathbf{z}_{v}\right)\right)+O\left(\mu^{-3}\right)
\end{aligned}
$$

The Nagar bias is defined as the expected value of the first two terms, and hence equals

$$
N_{\mathrm{TSLS}}(\beta, \mathbf{C}, \mathbf{W}, \boldsymbol{\Omega})=\frac{1}{\operatorname{tr} \mathbf{S}_{2}}\left(\operatorname{tr} \mathbf{S}_{12}-2 \mathbf{C}_{0}^{\prime} \mathbf{S}_{12} \mathbf{C}_{0}\right) \mu^{-2} .
$$

A.3.2 Proof of Theorems 1.2 and 1.3. We prove Theorem 1.3 first. We assume that $\mathbf{W}$ and $\boldsymbol{\Omega}$ are positive definite, so $\mathbf{S}$ and $\boldsymbol{\Sigma}$ are also positive definite. $\mathbf{S}_{12}$ is real valued but not necessarily symmetric. Note that

$$
\operatorname{tr} \mathbf{S}_{12}-2 \mathbf{C}_{0}^{\prime} \mathbf{S}_{12} \mathbf{C}_{0}=\operatorname{tr} \mathbf{S}_{12}^{\mathrm{sym}}-2 \mathbf{C}_{0}^{\prime} \mathbf{S}_{12}^{\mathrm{sym}} \mathbf{C}_{0},
$$

where $\mathbf{S}_{12}^{\mathrm{sym}}=\frac{1}{2}\left(\mathbf{S}_{12}+\mathbf{S}_{12}^{\prime}\right)$ is the symmetric part of $\mathbf{S}_{12}$. Write

$$
\boldsymbol{\Lambda}=\left[\begin{array}{cccc}
\lambda_{1} & 0 & \ldots & 0 \\
0 & \lambda_{2} & \ldots & 0 \\
0 & 0 & \ldots & \lambda_{K}
\end{array}\right]
$$

for the diagonal matrix of eigenvalues of $\mathbf{S}_{12}^{\text {sym }}$. Assume the eigenvalues are ordered, so $\lambda_{1} \geq \lambda_{2} \geq \cdots \geq \lambda_{K}$. For any real matrix $\mathbf{M}$, we write $|\mathbf{M}|=\sqrt{\mathbf{M}^{\prime} \mathbf{M}}$, so the Schatten 1-norm for matrices is defined as $\|\mathbf{M}\|_{1}=\operatorname{tr}|\mathbf{M}|$.

$$
\begin{aligned}
\operatorname{tr} \mathbf{S}_{12}^{\mathrm{sym}}-2 \mathbf{C}_{0}^{\prime} \mathbf{S}_{12}^{\mathrm{sym}} \mathbf{C}_{0} & \leq \sum_{k=1}^{K} \lambda_{k}-2 \lambda_{K} \\
& =\sum_{k=1}^{K-1} \lambda_{k}-\lambda_{K} \\
& \leq \sum_{k=1}^{K}\left|\lambda_{k}\right| \\
& =\left\|\mathbf{S}_{12}^{\mathrm{sym}}\right\|_{1} .
\end{aligned}
$$

Similarly $\operatorname{tr} \mathbf{S}_{12}^{\mathrm{sym}}-2 \mathbf{C}_{0}^{\prime} \mathbf{S}_{12}^{\mathrm{sym}} \mathbf{C}_{0} \geq-\left\|\mathbf{S}_{12}^{\mathrm{sym}}\right\|_{1}$. Hence, $\mid \operatorname{tr} \mathbf{S}_{12}^{\mathrm{sym}}-$ $2 \mathbf{C}_{0}^{\prime} \mathbf{S}_{12}^{\mathrm{sym}} \mathbf{C}_{0} \mid \leq\left\|0.5 \mathbf{S}_{12}+0.5 \mathbf{S}_{12}^{\prime}\right\|_{1} \leq\left\|\mathbf{S}_{12}\right\|_{1}$. The last step follows from the triangle inequality and from the fact that the eigenvalues of $\mathbf{S}_{12}^{\prime} \mathbf{S}_{12}$ and $\mathbf{S}_{12} \mathbf{S}_{12}^{\prime}$ are the same.

Now $\operatorname{tr}\left(\mathbf{S}_{12}^{\prime} \mathbf{S}_{2}^{-1} \mathbf{S}_{12}\right) \leq \operatorname{tr}\left(\mathbf{S}_{1}\right)$, see, for example, Theorem 7.14 in Zhang (2010). Applying the matrix trace Cauchy-Schwarz inequality (Liu and Neudecker 1995, Theorem 1):

$$
\begin{aligned}
\left\|\mathbf{S}_{12}\right\|_{1}^{2} & =\left(\operatorname{tr}\left|\mathbf{S}_{12}\right|\right)^{2} \\
& \leq \operatorname{tr} \mathbf{S}_{2} \operatorname{tr}\left(\left|\mathbf{S}_{12}\right|^{\prime} \mathbf{S}_{2}^{-1}\left|\mathbf{S}_{12}\right|\right) \\
& =\operatorname{tr} \mathbf{S}_{2} \operatorname{tr}\left(\mathbf{S}_{12}^{\prime} \mathbf{S}_{2}^{-1} \mathbf{S}_{12}\right) .
\end{aligned}
$$

Putting this together, we get $\left\|\mathbf{S}_{12}\right\|_{1} \leq \sqrt{\operatorname{tr} \mathbf{S}_{1} \operatorname{tr} \mathbf{S}_{2}}$, proving Theorem 1.3.

The TSLS part of Theorem 1.2 follows from Theorem 1.3. For the LIML part note that $B_{\text {LIML }}(\mathbf{W}, \boldsymbol{\Omega})=\sup _{\beta \in \mathbb{R}} g_{\text {LIML }}(\beta)$, where

$$
\begin{aligned}
g_{\text {LIML }}(\beta)= & \max \left(\left|\frac{\operatorname{tr} \mathbf{S}_{12}-\frac{\sigma_{12}}{\sigma_{1}^{2}} \operatorname{tr} \mathbf{S}_{1}-\operatorname{maxeval} \mathbf{M}_{\mathbf{B}}}{\sqrt{\operatorname{tr} \mathbf{S}_{1}} \sqrt{\operatorname{tr} \mathbf{S}_{2}}}\right|,\right. \\
& \left.\left|\frac{\operatorname{tr} \mathbf{S}_{12}-\frac{\sigma_{12}}{\sigma_{1}^{2}} \operatorname{tr} \mathbf{S}_{1}-\operatorname{mineval} \mathbf{M}_{\mathbf{B}}}{\sqrt{\operatorname{tr} \mathbf{S}_{1}} \sqrt{\operatorname{tr} \mathbf{S}_{2}}}\right|\right),
\end{aligned}
$$

where $\mathbf{M}_{\mathbf{B}}=\frac{1}{2}\left(2 \mathbf{S}_{12}-\frac{\sigma_{12}}{\sigma_{1}^{2}} \mathbf{S}_{1}\right)+\frac{1}{2}\left(2 \mathbf{S}_{12}-\frac{\sigma_{12}}{\sigma_{1}^{2}} \mathbf{S}_{1}\right)^{\prime}$ and

$$
g_{\text {LIML }}(\beta) \rightarrow \frac{\text { maxeval }_{2}}{\operatorname{tr} \mathbf{W}_{2}} \text { as } \beta \rightarrow \pm \infty
$$

For $\mathbf{W}$ and $\boldsymbol{\Omega}$ nonsingular $g_{\text {LIML }}$ is continuous in $\beta$ everywhere, and hence bounded.

\section{A.4 Proof of Lemma 2}

Assume that $\mathbf{W}$ and $\boldsymbol{\Omega}$ are nonsingular. We prove that the test that rejects if

$$
\widehat{F}_{\text {eff }}>c\left(\alpha, \widehat{\mathbf{W}}_{2}, B_{e}(\widehat{\mathbf{W}}, \widehat{\boldsymbol{\Omega}}) / \tau\right)
$$

is asymptotically valid, that is, its asymptotic size is at most $\alpha$.

Claim 1: The function $F_{\mathbf{C}, \mathbf{W}_{2}}^{-1}(\alpha)$ is continuous in $\left\{\mathbf{C}, \mathbf{W}_{2}\right\}$.

Proof: $\boldsymbol{\gamma}_{2}^{\prime} \boldsymbol{\gamma}_{2} / \operatorname{tr}\left(\mathbf{W}_{2}\right)$ is a continuous random variable with nonzero density on $\mathbb{R}_{+}$, and therefore $F_{\mathbf{C}, \mathbf{W}_{2}}^{-1}(\alpha)$ is strictly decreasing and continuous in $\alpha$ everywhere. By Van der Vaart (2000, Lemma 21.2), the quantile function $F_{\mathbf{C}, \mathbf{W}_{2}}^{-1}(\alpha)$ is continuous in $\left\{\mathbf{C}, \mathbf{W}_{2}\right\}$ for any fixed $\alpha$.

Claim 2: The function $B_{e}(\mathbf{W}, \boldsymbol{\Omega})$ is lower semicontinuous.

Proof: The function $\left\|n_{e}\left(\beta, \mathbf{C}_{0}, \mathbf{W}, \boldsymbol{\Omega}\right)\right\| / \mathrm{BM}(\beta, \mathbf{W})$ is continuous in $\mathbf{W}$ and $\boldsymbol{\Omega} . B_{e}(\mathbf{W}, \boldsymbol{\Omega})$ is the supremum of continuous functions, and therefore is lower semicontinuous (Yeh 2000, p. 274).

Claim 3: The function $c\left(\alpha, \mathbf{W}_{2}, x\right)$ is lower semicontinuous in $\left\{\mathbf{W}_{2}, x\right\}$.

Proof: The function $\mathbb{1}_{\mathbf{C}^{\prime} \mathbf{C} / \operatorname{tr}\left(\mathbf{W}_{2}\right)<x}$ is an indicator function of an open set, and therefore lower semicontinuous in $\left\{\mathbf{W}_{2}, x\right\}$. The function $F_{\mathbf{C}, \mathbf{W}_{2}}^{-1}(\alpha)$ is continuous in $\mathbf{W}_{2}$ and greater than 0 . Hence, the product $F_{\mathbf{C}, \mathbf{W}_{2}}^{-1}(\alpha) \mathbb{1}_{\mathbf{C}^{\prime} \mathbf{C} / \operatorname{tr}\left(\mathbf{W}_{2}\right)<x}$ is lower semicontinuous in $\left(\mathbf{W}_{2}, x\right)$ for any fixed $\alpha . c\left(\alpha, \mathbf{W}_{2}, x\right)$ is a supremum of lower semicontinuous functions, and therefore lower semicontinuous in $\left(\mathbf{W}_{2}, x\right)$ (Yeh 2000, p. 274). $c\left(\alpha, \mathbf{W}_{2}, x\right)$ is also clearly nondecreasing in $x$.

Proof of Result: From the lower semicontinuity of $B(\mathbf{W}, \boldsymbol{\Omega})$ and the continuous mapping theorem, it follows that $\min \left(B_{e}(\widehat{\mathbf{W}}, \widehat{\boldsymbol{\Omega}}), B_{e}(\mathbf{W}, \boldsymbol{\Omega})\right) \stackrel{p}{\rightarrow} B_{e}(\mathbf{W}, \boldsymbol{\Omega})$. Similarly, for any $\left(\widehat{\mathbf{W}}_{2}, \widehat{x}\right) \stackrel{p}{\rightarrow}\left(\mathbf{W}_{2}, x\right)$, the continuous mapping theorem implies 
that $\min \left(c\left(\alpha, \widehat{\mathbf{W}}_{2}, \widehat{x}\right), c\left(\alpha, \mathbf{W}_{2}, x\right)\right) \stackrel{p}{\rightarrow} c\left(\alpha, \mathbf{W}_{2}, x\right)$. Then

$$
\begin{aligned}
& \mathbb{P}\left(\widehat{F}_{\text {eff }}>c\left(\alpha, \widehat{\mathbf{W}}_{2}, B_{e}(\widehat{\mathbf{W}}, \widehat{\boldsymbol{\Omega}}) / \tau\right)\right. \\
\leq & \mathbb{P}\left(\widehat{F}_{\text {eff }}>\min \left(c\left(\alpha, \mathbf{W}_{2}, \frac{B_{e}(\mathbf{W}, \boldsymbol{\Omega})}{\tau}\right),\right.\right. \\
& \left.\left.c\left(\alpha, \widehat{\mathbf{W}}_{2}, \frac{\min \left(B_{e}(\mathbf{W}, \boldsymbol{\Omega}), B_{e}(\widehat{\mathbf{W}}, \widehat{\boldsymbol{\Omega}})\right)}{\tau}\right)\right)\right) \\
\rightarrow & \mathbb{P}\left(\widehat{F}_{\text {eff }}^{*}>c\left(\alpha, \mathbf{W}_{2}, \frac{B_{e}(\mathbf{W}, \boldsymbol{\Omega})}{\tau}\right)\right) \\
= & \alpha
\end{aligned}
$$

Now we prove the second part of the Lemma. We first prove a bound for the critical values. Let $F_{\chi_{d}^{2}(x)}^{-1}(\alpha)$ the upper $\alpha$ point of a noncentral $\chi^{2}$ with $d$ degrees of freedom and noncentrality parameter $x$. For any $\alpha \in[0,1], c\left(\alpha, \mathbf{W}_{2}, x\right) \leq x^{*} \equiv$ $\left(\sqrt{\max \left(F_{\chi_{1}^{2}(0)}^{-1}(\alpha), F_{\chi_{2}^{2}(0) / 2}^{-1}(\alpha), \ldots, F_{\chi_{K}^{2}(0) / K}^{-1}(\alpha)\right)}+\sqrt{x}\right)^{2}$.

Let $X_{i} \sim N(0,1)$ iid, $i=1,2, \ldots, K$, and let $c \in A$, where $A=\left\{c \in \mathbb{R}^{K} \mid \sum_{i=1}^{K} c_{i}=1, c_{i} \geq 0, \forall i\right\}$. From Szekely and Bakirov (2003), $\tilde{x} \in \mathbb{R}_{+}$that $\inf _{c \in A} P\left(\sum_{i=1}^{K} c_{i} X_{i}^{2} \leq \tilde{x}\right)=$ $P\left(\chi_{n}^{2} / n(\tilde{x}) \leq \tilde{x}\right)$, where the function $n(\tilde{x})$ is integer, nondecreasing, bounded by $K$ and equal to 1 whenever $\tilde{x}>$ 1.536. Let $Q=\sum_{i=1}^{K} c_{i}\left(X_{i}+b_{i}\right)^{2}$ a quadratic form in normal random variables and write $\sum_{i=1}^{K} c_{i} b_{i}^{2}=\mu^{2}$. From the triangle inequality,

$$
\begin{aligned}
\mathbb{P}[Q>x] & =\mathbb{P}\left[\sum_{i=1}^{K} c_{i}\left(X_{i}+b_{i}\right)^{2}>x\right] \\
& \leq \mathbb{P}\left[\left(\sqrt{\sum_{i=1}^{K} c_{i} X_{i}^{2}}+\mu\right)^{2}>x\right] .
\end{aligned}
$$

Whenever $\quad x>\mu^{2} \quad$ then $\mathbb{P}[Q>x] \leq \mathbb{P}\left[\chi_{n\left(x_{1}\right)}^{2} / n\left(x_{1}\right)>\right.$ $\left.x_{1}\left(\mu^{2}, x\right)\right]$, where $x_{1}\left(\mu^{2}, x\right)=\left(x^{1 / 2}-\mu\right)^{2}$. Moreover, this bound is increasing in $\mu^{2}$ whenever $x>\mu^{2}$. Let $x^{*}$ as above. Then $x_{1}\left(x, x^{*}\right)=\max \left(F_{\chi_{1}^{2}(0)}^{-1}(\alpha), F_{\chi_{2}^{2}(0) / 2}^{-1}(\alpha), \ldots, F_{\chi_{K}^{2}(0) / K}^{-1}(\alpha)\right)$. Therefore, for $\mu^{2} \leq x$

$$
P\left[Q>x^{*}\right] \leq \mathbb{P}\left(\left[\chi_{n\left(x_{1}\right)}^{2} / n\left(x_{1}\right)>x_{1}\left(x, x^{*}\right)\right] \leq \alpha .\right.
$$

Now assume that $B_{e}(\widehat{\mathbf{W}}, \widehat{\boldsymbol{\Omega}})$ is bounded in probability. Then $c\left(\alpha, \widehat{\mathbf{W}}_{2}, B_{e}(\widehat{\mathbf{W}}, \widehat{\boldsymbol{\Omega}})\right)$ is bounded above in probability by some $c^{*}$. Then

$$
\begin{aligned}
\min \left[\mathbb{P}\left(\widehat{F}_{\text {eff }}>c\left(\alpha, \widehat{\mathbf{W}}_{2}, B_{e}(\widehat{\mathbf{W}}, \widehat{\boldsymbol{\Omega}}) / \tau\right)\right)\right), \\
\left.\mathbb{P}\left(\widehat{F}_{\text {eff }}>c^{*}\right)\right] \stackrel{p}{\rightarrow} \mathbb{P}\left(F_{\text {eff }}^{*}>c^{*}\right) .
\end{aligned}
$$

But then by the triangle inequality

$$
\mathbb{P}\left(F_{\text {eff }}^{*}>c^{*}\right) \geq \mathbb{P}\left(\mu>\sqrt{c^{*}}+\sqrt{\sum_{i=1}^{K} c_{i} X_{i}^{2}}\right),
$$

where $c_{i}$ are the eigenvalues of $\mathbf{W}_{2}$ and $X_{i}$ are iid standard normal. The right-hand side in (A.17) clearly converges to 1 as $\mu^{2} \rightarrow \infty$, proving the second part of the Lemma.

\section{SUPPLEMENTARY MATERIALS}

- [A Robust Test for Weak Instruments: Supplementary Materials.]. Computational details and additional results. (PDF)

- [Files201200717.zip] MATLAB and STATA code to compute figures, tables and critical values. (Zip file)

\section{ACKNOWLEDGMENTS}

The authors are grateful to John Campbell, Matias Cattaneo, Gary Chamberlain, Eduardo Davila, Joshua Gottlieb, Stephanie Hurder, Rustam Ibragimov, Guido Imbens, Michal Kolesar, Ignacio Lobato, Joshua Mendel, Marcelo Moreira, Daniel Pollmann, Doug Staiger, Luis Viceira, the editors Keisuke Hirano and Jonathan Wright, an anonymous associate editor, two anonymous referees, and seminar participants at the Harvard Econometrics Lunch and at the North American Summer Meeting of the Econometric Society 2011 for helpful comments and suggestions. The authors are especially grateful to Jim Stock for his invaluable advice and guidance. The usual disclaimer applies.

\section{[Received February 2011. Revised August 2012.]}

\section{REFERENCES}

Andrews, D. W. K., and Stock, J. H. (2006), "Inference With Weak Instruments," in Advances in Economics and Econometrics, Theory and Applications (9th Congress of the Econometric Society) (Vol. 3), eds. R. Blundell, W. K. Newey, and T. Persson, Cambridge: Cambridge University Press, Chapter 6, pp. 122-173. [359]

Antoine, B., and Lavergne, P. (2012), "Conditional Moment Models Under Semi-Strong Identification,” Mimeo, Simon Fraser University. [358]

Bansal, R., and Yaron, A. (2004), "Risks for the Long Run: A Potential Resolution of Asset Pricing Puzzles," Journal of Finance, 59, 1481-1509. [364]

Baum, C. F., Schaffer, M. E., and Stillman, S. (2007), "Enhanced Routines for Instrumental Variables/Generalized Method of Moments Estimation and Testing," The Stata Journal, 7, 465-506. [358]

Bun, M., and de Haan, M. (2010), "Weak Instruments and the First Stage F-statistic in IV Models With a Nonscalar Error Covariance Structure," Mimeo, University of Amsterdam, available at http://wwwl.feb.uva.nl pp/bin/1156fulltext.pdf. [359]

Campbell, J. (2003), "Consumption-Based Asset Pricing," Handbook of the Economics of Finance, 1, 803-887. [358,364]

Campbell, J., and Mankiw, N. (1989), "Consumption, Income and Interest Rates: Reinterpreting the Time Series Evidence," in NBER Macroeconomics Annual 1989 (Vol. 4), eds. O. J. Blanchard and S. Fischer, Cambridge, MA: MIT Press, pp. 185-246. [364]

Chao, J. C., Swanson, N. R., Hausman, J. A., and Newey, W. K. (2012), “Asymptotic Distribution of JIVE in the Heteroskedastic IV Regression With Many Instruments," Econometric Theory, 28, 42-86. [358]

Conerly, M. D., and Mansfield, E. R. (1988), "An Approximate Test for Comparing Heteroscedastic Regression Models," Journal of the American Statistical Association, 83, 811-817. [363]

Epstein, L., and Zin, S. (1989), "Substitution, Risk Aversion, and the Temporal Behavior of Consumption and Asset Returns: A Theoretical Framework,' Econometrica, 57, 937-969. [364]

(1991), "Substitution, Risk Aversion, and the Temporal Behavior of Consumption and Asset Returns: An Empirical Analysis," Journal of Political Economy, 99, 263-286. [364]

Farebrother, R. (1990), "Algorithm AS 256: The Distribution of a Quadratic Form in Normal Variables," Applied Statistics, 39, 294-309. [364]

Ghosh, B. (1973), "Some Monotonicity Theorems for $\chi^{2}, F$ and $t$ Distributions With Applications," Journal of the Royal Statistical Society, Series B, 35, 480-492. [364]

Grubbs, F. E. (1964), "Approximate Circular and Noncircular Offset Probabilities of Hitting," Operations Research, 12, 51-62. [363]

Guggenberger, P. (2010a), "The Impact of a Hausman Pretest on the Asymptotic Size of a Hypothesis Test," Econometric Theory, 26, 369-382. [359] 
(2010b), "The Impact of a Hausman Pretest on the Size of a Hypothesis Test: The Panel Data Case," Journal of Econometrics, 156, 337-343. [359]

Hall, R. E. (1988), "Intertemporal Substitution in Consumption," The Journal of Political Economy, 96, 339-357. [364]

Hansen, L., and Singleton, K. (1983), "Stochastic Consumption, Risk Aversion, and the Temporal Behavior of Asset Returns," The Journal of Political Economy, 91, 249-265. [364]

Hausman, J. A., Newey, W. K., Woutersen, T., and Chao, J. C. (2012), "Instrumental Variable Estimation With Heteroskedasticity and Many Instruments," Quantitative Economics, 3, 211-255. [358]

Henshaw, R. C. (1966), "Testing Single-Equation Least Squares Regression Models for Autocorrelated Disturbances," Econometrica, 34, 646-660. [363]

Imhof, J. (1961), "Computing the Distribution of Quadratic Forms in Normal Variables," Biometrika, 48, 419. [363,364]

Johnson, N. L., Kotz, S., and Balakrishnan, N. (1995), Continuous Univariate Distributions (Vol. 2), New York: Wiley. [363]

Kleibergen, F. (2007), "Generalizing Weak Instrument Robust IV Statistics towards Multiple Parameters, Unrestricted Covariance Matrices and Identification Statistics," Journal of Econometrics, 139, 181-216. [359]

Koerts, J., and Abrahamse, A. (1969), On the Theory and Application of the General Linear Model, Rotterdam: Rotterdam University Press. [364]

Leeb, H., and Poetscher, B. M. (2005), "Model Selection and Inference: Facts and Fiction," Econometric Theory, 21, 21-59. [359]

Liu, H., Tang, Y., and Zhang, H. (2009), "A New Chi-Square Approximation to the Distribution of Non-Negative Definite Quadratic Forms in Non-Central Normal Variables," Computational Statistics \& Data Analysis, 53, 853-856. [363]

Liu, S., and Neudecker, H. (1995), "Matrix-Trace Cauchy-Schwarz Inequalities and Applications in Canonical Correlation Analysis," Statistical Papers, 36, 287-298. [363,367]

Mikusheva, A. (2010), "Robust Confidence Sets in the Presence of Weak Instruments," Journal of Econometrics, 157, 236-247. [359]

Müller, U. K. (2011), "Efficient Tests Under a Weak Convergence Assumption," Econometrica, 79, 395-435. [359,362]

Nagar, A. (1959), "The Bias and Moment Matrix of the General k-Class Estimators of the Parameters in Simultaneous Equations," Econometrica, 27, 575-595. [358,362,366]
Patnaik, P. (1949), "The Non-Central Chi-Square and F-Distributions and Their Applications," Biometrika, 36, 202-232. [358,360,363]

Pearson, E. (1959), "Note on an Approximation to the Distribution of NonCentral Chi-Squared," Biometrika, 46, 364. [363]

Rothenberg, T. J. (1984), "Approximating the Distributions of Econometric Estimators and Test Statistics," in Handbook of Econometrics (Vol. 2), eds. Z. Griliches and M. D. Intriligator, Amsterdam: North-Holland, Chapter 15, pp. 881-935. [363,366]

Ruud, P. (2000), An Introduction to Classical Econometric Theory, New York: Oxford University Press. [364]

Satterthwaite, F. E. (1946), "An Approximate Distribution of Estimates of Variance Components," Biometrics Bulletin, 2, 110-114. [363]

Staiger, D., and Stock, J. (1997), "Instrumental Variables Regression With Weak Instruments," Econometrica, 65, 557-586. [358,359,362]

Stock, J., Wright, J., and Yogo, M. (2002), "A Survey of Weak Instruments and Weak Identification in Generalized Method of Moments," Journal of Business \& Economic Statistics, 20, 518-529. [359]

Stock, J., and Yogo, M. (2005), "Testing for Weak Instruments In Linear IV Regression," in Identification and Inference for Econometric Models: Essays in Honor of Thomas Rothenberg, eds. D. W. Andrews and J. H. Stock. Cambridge: Cambridge University Press, pp. 80-108. [358,359,362,364,365]

Szekely, G., and Bakirov, N. (2003), "Extremal Probabilities for Gaussian Quadratic Forms," Probability Theory and Related Fields, 126, 184-202. [368]

Theil, H., and Nagar, A. (1961), "Testing the Independence of Regression Disturbances," Journal of the American Statistical Association, 56, 793806. [363]

Van der Vaart, A. (2000), Asymptotic Statistics, Cambridge: Cambridge University Press. [367]

Yeh, J. (2000), Lectures on Real Analysis, Singapore: World Scientific. [367]

Yogo, M. (2004), "Estimating the Elasticity of Intertemporal Substitution When Instruments are Weak," Review of Economics and Statistics, 86, 797-810. $[358,364]$

Zhan, Z. (2010), “Detecting Weak Identification by Bootstrap,” Mimeo, Brown University. [359]

Zhang, F. (2010), Matrix Theory: Basic Results and Techniques (2nd ed.), New York: Springer. [367] 